\title{
CoREST has a conserved role in facilitating SPR-5/LSD1 maternal reprogramming of histone methylation
}

Brandon S. Carpenter ${ }^{1, \#}$, Alyssa Scott1,\#, Robert Goldin ${ }^{2}$, Sindy R. Chavez ${ }^{1}$, Dexter A. Myrick ${ }^{1}$, Marcus Curlee ${ }^{3}$, Karen Schmeichel ${ }^{4}$, David J. Katz ${ }^{1^{*}}$

1Department of Cell Biology, Emory University School of Medicine, Atlanta GA 30322, USA.

${ }^{2}$ Uniformed Services University School of Medicine, Bethesda MD 20814, USA.

${ }^{3}$ Department of Cell and Developmental Biology, Perelman School of Medicine, University of Pennsylvania, Philadelphia PA 19104, USA.

${ }^{4}$ Natural Sciences Division, Oglethorpe University, Atlanta GA 30319, USA.

\#Authors contributed equally

*corresponding author:

David J. Katz

Associate Professor

Department of Cell Biology

Room 443, Whitehead Biomedical Research Building

Emory University School of Medicine

Atlanta, GA 30322, USA

Phone: (404) 727-3403

djkatz@emory.edu 


\begin{abstract}
Maternal reprogramming of histone methylation is critical for reestablishing totipotency in the zygote, but how histone modifying enzymes are regulated during maternal reprogramming is not well characterized. To address this gap, we asked whether maternal reprogramming by the H3K4me1/2 demethylase SPR-5/LSD1/KDM1A, is regulated by the co-repressor protein, SPR-1/CoREST in C. elegans and mice. In C. elegans, SPR-5 functions as part of a reprogramming switch together with the H3K9 methyltransferase MET-2. By examining germline development, fertility and gene expression in double mutants between $s p r-1$ and met-2, we find that spr-1 mutants are partially compromised for spr-5; met-2 reprogramming. In mice, we generated a separation of function Lsd1 M448V point mutation that compromises CoREST binding, but only slightly affects LSD1 demethylase activity. When maternal LSD1 in the oocyte is derived exclusively from this allele, the progeny phenocopy the increased perinatal lethality that we previously observed when LSD1 was reduced maternally. Together, these data are consistent with CoREST having a conserved function in facilitating maternal LSD1 epigenetic reprogramming.
\end{abstract}

\title{
INTRODUCTION
}

Re-establishing the transcriptional ground state to enable embryonic development in a newly formed zygote requires extensive maternal reprogramming of chromatin at fertilization (Burton and Torres-Padilla, 2014; Hemberger et al., 2009; Li, 2002; Morgan et al., 2005; Seisenberger et al., 2012). Maternal reprogramming of chromatin is accomplished by the deposition of enzymes into the oocyte that covalently modify histones. The 
combination of these histone modifications contributes to developmental cell fates by regulating the accessibility of chromatin for transcription. For example, methylation of either lysine 9 or 27 on histone H3 (H3K9me and H3K27me) is generally associated with repressed transcription, whereas methylation of either lysine 4 or 36 on histone H3 (H3K4me and H3K36me) is associated with active transcription (Bannister et al., 2005; Barski et al., 2007; Bernstein et al., 2002; Bernstein et al., 2005).

Accumulating evidence suggests that patterns of histone modifications can be maintained through cell divisions to help maintain cell fate. For example, during early patterning in Drosophila, the expression of homeotic genes is modulated by segmentation transcription factors. After the segmentation factors turn over, the continued maintenance of homeotic gene expression through development is dependent on the H3K27 and H3K4 methyltransferases, Polycomb and Trithorax (Coleman and Struhl, 2017; Moehrle and Paro, 1994; Simon and Tamkun, 2002). Likewise, in C. elegans, the Polycomb Repressive Complex 2 (PRC2), which includes MES-2/3/6, maintains paternally inherited H3K27me3 during embryogenesis (Gaydos et al., 2014; Kaneshiro et al., 2019; Tabuchi et al., 2018). In addition to H3K27me3, the maternally deposited H3K36 methyltransferase, MES-4, maintains $\mathrm{H} 3 \mathrm{~K} 36 \mathrm{me} 2 / 3$ at a subset of germline genes (MES-4 germline genes) in a transcriptionally independent manner to help reestablish the germline in the next generation (Furuhashi et al., 2010; Rechtsteiner et al., 2010). Furthermore, the transgenerational inheritance of repressive histone modifications occurs in C. elegans mutants lacking the COMPASS complex component, WDR-5. wdr-5 mutants transgenerationally extend lifespan due to the accumulation of H3K9me2 across generations (Greer et al., 2010; Lee et al., 2019). Histone methylation may also be 
transmitted across generations in vertebrates (Brykczynska et al., 2010; Hammoud et al., 2009; Wu et al., 2011; Zheng et al., 2016; Zhu et al., 2019). Together these findings suggest that inherited histone methylation patterns are conserved across multiple phyla, and that the inheritance of the proper chromatin state is critical for normal function of the offspring.

Despite the importance of inherited histone methylation in contributing to the maintenance of cell fates, there are developmental transitions where the inheritance of histone methylation may need to be prevented. For example, in C. elegans, the histone demethylase SPR-5, must remove H3K4me1/2 at fertilization to prevent the inappropriate inheritance of previously specified transcriptional states. Failure to erase H3K4me1/2 at fertilization between generations in spr-5 mutants correlates with an accumulation of H3K4me2 and ectopic spermatogenesis gene expression across $\sim 30$ generations. This accumulation of H3K4me2 leads to progressively increasing sterility, which is defined as germline mortality (Katz et al., 2009). SPR-5 reprogramming at fertilization functions together with the addition of H3K9 methylation by the methyltransferase MET-2 (Greer et al., 2014; Kerr et al., 2014). spr-5; met-2 mutants have a synergistic sterility where progeny are sterile in a single generation, rather than over many generations (Kerr et al., 2014). Together this work supports a model in which SPR-5 and MET-2 are maternally deposited into the oocyte, where they reprogram histone methylation at fertilization to prevent defects caused by inappropriately inherited transcriptional states. Furthermore, in $C$. elegans the transgenerational maintenance of H3K36me3 by MES-4 functions to antagonize SPR-5/MET-2 repression, enabling the proper specification of the germline in the progeny (Carpenter et al., 2021). 
SPR-5/MET-2 epigenetic reprogramming at fertilization is conserved in mammals. When the met-2 ortholog Setdb1 is maternally deleted in mice, zygotes develop slowly and die by the blastocyst stage (Eymery et al., 2016; Kim et al., 2016). Similarly, when the spr-5 ortholog Lsd1 is maternally deleted in mice, embryos die at the 1-2 cell stage, and these mutants are more transcriptionally similar to an oocyte than a wild type 1-2 cell embryo (Ancelin et al., 2016; Wasson et al., 2016). Thus, without maternally provided LSD1, embryos are unable to undergo the maternal-to-zygotic transition. Moreover, when maternal LSD1 protein levels are decreased, some animals can bypass the 1-2 cell arrest and survive until birth. However, progeny that are born exhibit lethality shortly after birth (perinatal lethal), indicating that incomplete reprogramming at fertilization can have phenotypes that manifest postnatally (Wasson et al., 2016). The 1-2 cell arrest and perinatal lethality phenotypes potentially occur through the inappropriate inheritance of histone methylation.

Although the evidence for maternal reprogramming across multiple taxa is mounting, it is not clear how histone modifying enzymes like LSD1 are regulated during this process. Recently, studies have implicated the co-repressor CoREST in broadly regulating LSD1 function. In mice, LSD1 and CoREST are often found in the same transcriptional corepressor complex together (Hakimi et al., 2003; Humphrey et al., 2001; Shi et al., 2003; You et al., 2001). In addition, LSD1 and CoREST have been co-crystallized, (Yang et al., 2006) and CoREST is required for the stability of LSD1 (Foster et al., 2010; Shi et al., 2005). CoREST may also be required for full LSD1 function, because although LSD1 can demethylate H3K4 peptides or bulk histones in vitro, it is only capable of demethylating nucleosomes when in complex with CoREST (Lee et al., 2005; Shi et al., 2005; Yang et al., 
2006). Additionally, LSD1 and CoREST phenocopy each other in multiple organisms. In Drosophila, LSD1 and CoREST have overlapping functions in spermatogenesis and in ovary follicle progenitor cells (Lee and Spradling, 2014; Mačinković et al., 2019). In C. elegans, the homolog of CoREST, SPR-1, was identified, along with SPR-5, in a suppressor screen for the ability to rescue the egg-laying defect (Egl) associated with loss of SEL-12, a presenilin protein (Wen et al., 2000). Furthermore, SPR-1 has been shown to physically interact with SPR-5 in vitro and in vivo (Eimer et al., 2002; Kim et al., 2018). Together, these data raise the possibility that LSD1 could be functioning through CoREST to maternally reprogram histone methylation, but this hypothesis has not yet been tested.

Here, we utilize both mouse and C. elegans to test whether LSD1 and CoREST function together maternally. We demonstrate that C. elegans lacking SPR-1 display a reduction in brood size that is between wildtype and spr-5 mutants. Unlike spr-5 mutants, spr-1 mutants do not become increasingly sterile across $\sim 30$ generations. However, when maternal reprogramming is sensitized by loss of the met-2 gene, met-2; spr-1 mutants reveal intermediate sterility and gene expression changes that are exacerbated compared to single mutants, but less affected than spr-5; met-2 mutants. We also demonstrate that met-2; spr-1 mutants misexpress MES-4 germline genes in somatic tissues at intermediate levels compared to spr-5; met-2 mutants. In mice, we find that LSD1 and CoREST are both expressed in mouse oocyte nuclei. In addition, we generated a separation of function Lsd1 point mutation that compromises CoREST binding, but only slightly affects LSD1 demethylase activity. When this mutation is inherited maternally, the progeny phenocopy the increased perinatal lethality that we previously observed when LSD1 was reduced 
maternally. Together, these data are consistent with CoREST having a conserved function in facilitating maternal LSD1 epigenetic reprogramming.

\section{RESULTS}

\section{spr-1 mutants have reduced fertility but do not exhibit germline mortality}

Previously, we demonstrated that populations of spr-5 mutants become increasingly sterile over $\sim 30$ generations (Katz et al., 2009). Therefore, if SPR-1 is required for SPR-5 maternal reprogramming activity, it is possible that spr-1 mutants might phenocopy the germline mortality across generations observed in spr-5 mutants. To address this possibility, we performed germline mortality assays on wild type (Bristol N2 strain, hereafter referred to as wild type), spr-1 mutant, and spr-5 mutant animals (Fig. 1A). Wild type hermaphrodites gave rise to $\sim 300$ progeny in the first generation (F1) and this average number of progeny was maintained through 50 generations (Fig. 1A). Similar to what we previously reported, progeny from F1 spr-5 mutants average 150-200 progeny, and by generation 23 (F23), the average number of progeny declined to $\sim 60$ animals (Fig. 1A). spr-1 mutants averaged $\sim 250$ progeny in the first generation. This average number of progeny is intermediate between spr-5 mutants and wild type. But unlike spr-5 mutants, spr-1 mutants never became sterile across generations (Fig. 1A).

\section{met-2; spr-1 double mutants exhibit germline mortality}

Previously we demonstrated that SPR-5 synergizes with the H3K9me2 methyltransferase, MET-2, to regulate maternal epigenetic reprogramming (Kerr et al., 2014). Progeny of mutants lacking both SPR-5 and MET-2 are completely sterile in a single 
generation (Kerr et al., 2014). If SPR-1 is partially required for SPR-5 maternal reprogramming, then it is possible that spr-1 mutants will exhibit a synergistic phenotype when combined with a met-2 mutation. To determine whether spr-1 mutants display any abnormal phenotypes in a met-2 mutant background, we individually cloned out 288 progeny from first generation (F1) met-2; spr-1 double mutants and examined each animal for sterility (Fig. 1B-D). Of the 288 individually cloned met-2; spr-1 F1 mutant progeny, 208 were fertile (Fig. 1B,C) and 80 were sterile (Fig. 1D). We also observed that 76 of the 208 fertile F1 progeny die as young adults due to defects in egg laying (Fig. 1C). To determine whether fertile met-2; spr-1 mutants become germline mortal, we counted the average number of progeny from met-2; spr-1 mutants over successive generations and compared them to wild type, spr-1 and met-2 mutants (Fig. 1E; Fig. S1A,C). As observed previously, the average number of progeny from spr-1 and met- 2 mutants were lower than wild type, but remained consistent over 10 generations, and neither mutant gave rise to sterile animals over that time frame (Fig. 1E,F; Fig. S1A,B). Fertile met-2; spr-1 mutant progeny produced an average of $\sim 70$ progeny in the first generation. However, by generation 10 the average number of progeny declined to $\sim 30$ (Fig. 1E; Fig. S1C). Consistent with this germline mortality phenotype, the number of completely sterile animals in met-2; spr-1 mutants increased across successive generations from $\sim 30 \%$ at early generations to $\sim 60 \%$ by F10 (Fig. 1F; Fig. S1D). Together, these results show that met-2; spr-1 mutants display a germline mortality phenotype that is intermediate between the maternal effect sterility of spr-5; met-2 mutants in a single generation and the germline mortality of spr-5 and met-2 single mutants over $\sim 30$ generations. 


\section{The sterility of met-2; spr-1 mutants resembles spr-5; met-2 mutants}

We also examined the gonads of sterile met-2; spr-1 mutants to determine if the sterility resembles the sterility of $s p r-5$; met-2 mutants. spr-5; met-2 mutants have a squat germline, with both gonad arms failing to elongate (Fig. S2A,B; Kerr et al., 2014). Within the squat germline there are proliferating germ cells, sperm and oocytes, indicating that the germline has proceeded through normal transitions. However, these cell types are inappropriately interspersed (Carpenter et al., 2021; Katz et al., 2009). Unlike spr-5; met-2 mutants, F1 sterile met-2; spr-1 mutants have elongated gonad arms. However, within these sterile F1 gonads, we observed a similarly disorganized mixture of germ cells, sperm and oocytes (Fig. S2C,D). In addition, after several generations the germlines of sterile met-2; spr-1 mutants resembled the squat germlines of spr-5; met-2 mutants, although unlike spr5; met-2 mutants, some animals remained partially fertile at later generations (Fig. 1E; Fig. $\mathrm{S} 2 \mathrm{~A}, \mathrm{~B}, \mathrm{E}, \mathrm{F})$. Thus, the sterility of met-2; spr-1 mutants at late generations phenocopies the maternal effect sterility of spr-5; met-2 mutants observed in the first generation.

\section{Transcriptional misregulation in met-2; spr-1 progeny resembles that observed in}

\section{spr-5; met-2 progeny but is less affected}

Since the severity of the germline mortality phenotype of met-2; spr-1 mutants is between spr-5; met-2 mutants and spr-1 or met-2 single mutants, it raises the possibility that maternal SPR-5 reprogramming may be partially dependent upon the SPR-5 interacting partner SPR-1. If mutating spr-1 partially compromises SPR-5 maternal reprogramming, we would expect that the genes that are misexpressed in met-2; spr-1 mutants would be similar to spr-5; met-2 mutants, but that the gene expression changes 
would be less affected in met-2; spr-1 mutants. To test this possibility, we performed RNAseq on F7 spr-1, met-2, and met-2; spr-1 mutant L1 progeny compared to wild type L1 progeny. We chose to perform the analysis on F7 met-2; spr-1 mutants because this generation precedes the increase in sterility that we observed in our germline mortality assay after F7 (Fig. 1F; Fig. S1A). Thus, by performing the analysis at F7, it allowed us to observe primary effects from the loss of MET-2 and SPR-1, rather than secondary effects due to the sterility. In addition, we utilized starved L1 larvae for our RNA-seq analysis for two reasons. First, starved L1 larvae only have two germ cells that are not undergoing transcription. As a result, performing RNA-seq on these larvae allows us to exclusively examine somatic transcription. Second, we have previously performed RNA-seq and differential gene expression analysis on the L1 stage of spr-5; met-2 mutant progeny, so performing the RNA-seq analysis on met-2; spr-1 mutants at the L1 stage allows us to compare to our previously published data set (Carpenter et al., 2021). We identified 1,787 differentially expressed genes (DEGs) in met-2; spr-1 mutant progeny compared to wild type (Fig. S3A; Fig. S4C,F), and most of these genes are differentially expressed in met-2 (856/1327)(Fig. S3A,B; Fig. S4B,E) and spr-1 single mutants (40/60)(Fig. S3A,B; Fig. S4A,D) compared to wild type (Fig. S3B).

To determine whether gene expression changes in met-2; spr-1 mutants resemble those in our previously published spr-5; met-2 mutant RNA-seq data set, we first compared DEGs between the two data sets. We identified 1,787 DEGs in met-2; spr-1 mutant progeny compared to wild-type. 1,010 (57\%) of these significantly overlapped with the 4,223 DEGs that we previously identified in spr-5; met-2 mutant progeny compared to wild-type (Fig. 2A, hypergeometric test, P-value < 1.28E-270, (Carpenter et al., 2021), and the gene 
ontology categories of DEGs in both data sets are similar (Fig. S3C,D; Carpenter et al., 2021). We also examined the overlapped gene expression changes between up-regulated and down-regulated DEGs in both datasets separately. Of the 1,067 up-regulated DEGs in met-2; spr-1 mutant progeny, 676 (63\%) of these overlap with the 2,330 up-regulated DEGs in spr-5; met-2 mutant progeny (Fig. 2B, hypergeometric test, P-value < 2.61E-392; Carpenter et al., 2021). Of the 720 down-regulated DEGs in met-2; spr-1 mutant progeny, $236(33 \%)$ of these overlap with the 1,893 DEGs down-regulated DEGs in spr-5; met-2 mutant progeny (Fig. 2C, hypergeometric test, P-value < 2.16E-72; Carpenter et al., 2021).

If mutating spr-1 partially compromises SPR-5 maternal reprogramming, we would expect that the gene expression changes in met-2; spr-1 mutants would be less affected in spr-5; met-2 mutants. To determine if this is the case, we compared the average log2 fold change (FC) of the upregulated and downregulated DEGs separately. The average log2 (FC) of DEGs that are up-regulated in met-2; spr-1 mutant progeny is 2, compared to 3.1 in spr-5; met-2 mutant progeny (Fig. 3D; Carpenter et al., 2021). Similarly, the average log2(FC) of DEGs that are down-regulated in met-2; spr-1 mutant progeny is -1, compared to -1.4 in spr5; met-2 mutant progeny (Fig. 3E; Carpenter et al., 2021). Together, these data demonstrate while the same genes are differentially expressed in spr-5; met-2 and met-2; spr-1 mutant progeny, the changes are smaller in met-2; spr-1 mutant progeny.

\section{MES-4 germline genes are enriched in met-2; spr-1 mutants, but less affected}

\section{compared to spr-5; met-2 mutants}

MES-4 germline genes are genes that are expressed in the parental germline and acquire H3K36 methylation, which is maintained by a transcription independent 
methyltransferase MES-4 in the embryo of the progeny. Recently, we demonstrated that spr-5; met-2 mutants ectopically express 112 (57\%) out of the 196 MES-4 germline genes in somatic tissues (Carpenter et al., 2021). If the loss of SPR-1 partially compromises SPR-5 function, we would expect that MES-4 germline genes would also be ectopically expressed in met-2; spr-1 mutants, though to a lesser degree. Of 196 MES-4 germline genes, 45 (23\%) MES-4 germline genes were misexpressed in met-2; spr-1 mutant progeny compared to wild type (Fig. 3A, hypergeometric test, P-value < 1.41E-9). All of these $45 \mathrm{MES}-4$ germline genes overlap with the 112 MES-4 germline genes that are misregulated in spr-5; met-2 mutants (Fig. 3B; Carpenter et al., 2021). Thus, like spr-5; met-2 mutants, met-2; spr-1 mutants ectopically express MES-4 germline genes. However, when we compared the $\log 2$ (FC) in expression of all of the MES-4 germline genes in spr-1, met-2, met-2; spr-1, and spr-5; met-2 mutant progeny compared to wild type, we observed that the changes in the levels of gene expression are less affected in met-2; spr-1 mutants than spr-5; met-2 mutants (Fig. 3C; Carpenter et al., 2021).

\section{LSD1 and CoREST are expressed during each stage of mouse oocyte development}

Taken together, our results are consistent with SPR-5/LSD1 functioning maternally through SPR-1/CoREST in C. elegans. To determine whether there is a role for CoREST in LSD1 maternal reprogramming in mammals, we also sought to investigate the maternal interaction between LSD1 and CoREST in mice. Previous studies have shown that LSD1 is expressed during all stages of mouse oocyte development (Wasson et al. 2016, Ancelin et al. 2016, Kim et al. 2015). If LSD1 and CoREST function together in a complex, we would expect them to be expressed during the same stages of oogenesis. Previously CoREST was 
shown to be expressed in mouse oocytes, but the precise stages of oogenesis in which CoREST is expressed were not characterized (Ma et al., 2012). Thus, to determine whether CoREST is expressed at the same time as LSD1 in mouse oogenesis, we performed immunofluorescence experiments and examined CoREST and LSD1 protein at the primary, secondary, and antral stages of oocyte development (Fig. 4). Identical to what we and others previously observed with LSD1 (Fig. 4 A-I; Ancelin et al., 2016; Kim et al., 2015b; Wasson et al., 2016), CoREST was also highly expressed in the oocyte nucleus and in the surrounding follicle cells during all stages of oocyte development (Fig. 4 J-R).

\section{Reducing the function of maternally-provided LSD1 causes perinatal lethality}

Since CoREST has the same expression pattern as LSD1 in mouse oocytes, we wanted to test whether LSD1 functions through CoREST by specifically disrupting the presumptive CoREST-LSD1 interaction in the mouse oocyte. To do this, we utilized CRISPR to generate a point mutation, M448V, in the tower domain of the Lsd1 gene at the endogenous locus (Fig. 5A; Fig. S5). This allele will be referred to as $L s d 1^{M 448 V}$. The tower domain is the site of protein-protein interactions (Forneris et al., 2007; Stavropoulos et al., 2006; Yang et al., 2006), and M448V resides in a residue that binds CoREST (Shi et al., 2005). Previous studies have shown that this mutation reduces the ability of LSD1 to demethylate histones in vitro (85\% demethylase activity compared to wild-type LSD1) (Nicholson et al., 2013). This modest reduction in LSD1 function is unlikely to compromise maternal reprogramming. However, the M448V mutation severely reduces the ability of LSD1 to bind CoREST (35\% binding activity compared to wild type in vitro) (Nicholson et 
al., 2013). Thus, the M448V mutation serves as a separation-of-function allele between demethylase activity and CoREST binding.

To interrogate the interaction between LSD1 and CoREST specifically in oocytes, we utilized our newly generated M448V Lsd1 mutation. Previous studies have shown that a complete loss of LSD1 protein in the mouse oocyte results in embryonic arrest of offspring at the 1-2 cell stage (Ancelin et al., 2016; Wasson et al., 2016). This arrest is due to a failure to undergo the maternal-to-zygotic transition in gene expression. When LSD1 protein levels are decreased in the mouse oocyte, embryos can bypass the embryonic arrest, but $\sim 30 \%$ of animals die perinatally, shortly after birth (Wasson et al., 2016). Our results in C. elegans suggest that loss of CoREST results in a partial loss of LSD1 function. Therefore, if LSD1 function partially requires CoREST maternally in mice, we would expect that having only the Lsd $1^{M 448 V}$ allele maternally would result in offspring that phenocopy the perinatal lethality observed from a partial loss of maternal LSD1. To test this possibility, we generated mice with the $L s d 1^{M 448 V}$ allele over a floxed allele of the $L s d 1$ gene. In the presence of an oocytespecific Zp3-Cre allele that expresses prior to the first meiotic division, the floxed allele recombines to a null allele in the oocyte. As a result, the only maternal contribution of LSD1 is from the $L s d 1^{M 448 V}$ allele, which produces LSD1 with a reduced ability to bind CoREST (Fig. 5B). The F1 offspring from this cross will be referred to as $L s d 1^{M 448 V}$ progeny. Importantly, the mothers have a normal copy of the Lsd1 gene in every other cell type throughout the mouse, and heterozygous Lsd1 animals have been shown to have no phenotypic defects (Engstrom et al., 2020; Foster et al., 2010; Jin et al., 2013; Wang et al., 2007). In addition, mothers with a compromised maternal Lsd1 allele are crossed to wild-type males, so the progeny have normal zygotic LSD1 activity from their paternal allele after transcription 
begins at the 2-cell stage. This mating scheme enables us to determine the specific effect of compromising LSD1 activity maternally in the oocyte. In one set of controls, the mother has the $L s d 1^{M 448 V}$ allele over a floxed allele of LSD1 and is Zp3Cre negative (Fig. 5C). The maternal contribution in this case would be one functional copy of LSD1 and one hypomorphic Lsd1 ${ }^{M 448 V}$ copy. These control F1 offspring will be referred to as $L s d 1^{+}$. In the other set of controls, the mother has a wild-type copy of the Lsd1 gene over a floxed allele of $L s d 1$, and are Zp3Cre positive (Fig. 5D). The maternal contribution will be just one functional copy of the $L s d 1$ gene. Previous studies have shown animals that are heterozygous for the Lsd1 null allele have $70 \%$ protein levels (30\% reduction) compared to homozygotes (Engstrom et al., 2020). These control F1 offspring will be referred to as Lsd1 het.

If disrupting the interaction between LSD1 and CoREST specifically in the oocyte phenocopies the perinatal lethality that we previously observed when LSD1 is hypomorphic maternally, it would provide further evidence that LSD1 functions in a complex with CoREST in the oocyte. To address this possibility, we examined perinatal lethality between postnatal day $0(\mathrm{P} 0)$ and $\mathrm{P} 1$ in $L s d 1^{M 448 V}$ progeny versus $L s d 1^{+}$and $L s d 1^{\text {het }}$ controls. All litters were generated from mothers that were less than 8 months old, to avoid any complications associated with advanced maternal age. Overall, we observe increasing perinatal lethality with increasingly compromised maternal LSD1. In $L s d 1^{+}$control progeny, when one allele of Lsd1 lacks the ability to bind CoREST, we observe 9\% (N=24) perinatal lethality during the first $48 \mathrm{hrs}$ after birth. When one copy of $L s d 1$ is fully deleted maternally (Lsd1 het progeny), the perinatal lethality increased to $18 \%(\mathrm{~N}=15)$, and when maternal LSD1 is solely provided from the $\operatorname{Lsd1} 1^{M 48 V}$ allele, perinatal lethality further increases to 35\% (N=32)(Fig. 6E). The 35\% perinatal lethality, when LSD1 completely lacks 
the ability to bind CoREST maternally, is similar to the $\sim 30 \%$ perinatal lethality that we previously observed when LSD1 is partially lost maternally (Wasson et al., 2016). Importantly, the level of perinatal lethality does not depend on which allele the pup inherits from its mother (Fig. S6). Moreover, the entire litter died in 10 litters of Lsd1 $1^{\text {M48BV }}$ progeny out of 32 total (31.2\%), versus only 2 litters out of $14(14.2 \%)$ in Lsd 1 het animals, and 0 out of 24 litters (0\%) in $L s d 1^{+}$controls (Supplemental file 1 ). The $31 \%$ of $L s d 1^{\text {M448V }}$ litters in which all of the animals within the litter die is similar to the 10 out of 20 litters $(50 \%)$ in which the entire litter died that we previously observed upon partial loss of maternal LSD1 (Wasson et al., 2016). Thus, the loss of LSD1's ability to bind CoREST maternally phenocopies the perinatal lethality observed in progeny from mothers with partial loss of LSD1 protein in the oocyte.

\section{DISCUSSION}

\section{CoREST regulates LSD1 maternal reprogramming of histone methylation}

Despite our increasing knowledge of the enzymes involved in maternal epigenetic reprogramming, how these enzymes are regulated remains unclear. To begin to address this question, we asked whether maternal epigenetic reprogramming by the H3K4me1/2 demethylase SPR-5/LSD1/KDM1A is dependent on SPR-1/CoREST. In C. elegans, we find that the fertility of $s p r-1$ mutants is intermediate between spr-5 mutants and wild type, which raises the possibility that loss of the spr-1 gene partially compromises SPR-5 maternal reprogramming. However, spr-1 mutants do not phenocopy the germline mortality phenotype of $s p r-5$ mutants. This suggests that if SPR-1 contributes to SPR-5 reprogramming, SPR-5 function is not completely dependent upon SPR-1. 
Because SPR-5 and the H3K9 methyltransferase MET-2 function together in maternal reprogramming (Carpenter et al., 2021; Kerr et al., 2014; Greer et al., 2014), it provides a unique opportunity to ask whether SPR-1 functions in maternal SPR-5 reprogramming by making double mutants between spr-1 and met-2. If loss of SPR-1 partially compromises SPR-5 reprogramming, then spr-1 mutants might also display a synergistic sterility phenotype when combined with a mutation in met-2. Consistent with this possibility, met-2; spr-1 double mutants had a germline mortality phenotype that is intermediate between the maternal effect sterility of spr-5; met-2 mutants and the germline mortality of spr-5 and met-2 single mutants. To determine if this synergistic sterility phenotype is due to loss of SPR-1 partially compromising SPR-5 reprogramming, we performed two additional experiments. First, we examined the gonads of met-2; spr-1 double mutants to determine if the germline phenotype resembles the germline phenotype of spr-5; met-2 mutants. This analysis demonstrated that the germline phenotype of met-2; spr-1 mutants at late generations, with a squat gonad and disorganized germline cell types, is similar to spr-5; met-2 mutants. This is consistent with the possibility that loss of SPR-1 partially compromises SPR-5 reprogramming. Second, we performed RNA-seq on F7 met-2; spr-1 mutants. If SPR-1 functions specifically with SPR-5, we would expect that the genes that are misexpressed in met-2; spr-1 mutants to be similar to the genes that are affected in spr-5; met-2 mutants. Consistent with this possibility we observe a significant overlap in differentially expressed genes between met-2; spr-1 mutants and spr-5; met-2 mutants. In addition, the gene expression pathways affected in met-2; spr-1 mutants are similar to those affected in spr-5; met-2 mutants. However, if loss of SPR-1 only partially compromises SPR-5 function, we would expect that the magnitude of the gene expression 
changes in met-2; spr-1 mutants would be less changed than in spr-5; met-2 mutants.

Strikingly, we find that the gene expression changes in met-2; spr-1 mutants are consistently less affected than those that we observed previously in spr-5; met-2 mutants.

MES-4 germline genes become ectopically expressed in the absence of SPR-5/MET-2 reprogramming. If SPR-1 partially compromises maternal reprogramming, we would expect that MES-4 germline genes would also be ectopically expressed in the soma of met2; spr-1 mutants. The starved L1 larvae that we used for met-2; spr-1 mutant RNA-seq only have two germ cells that are not undergoing transcription. Despite this, we observe the expression of MES-4 germline genes in L1 met-2; spr-1 mutants. This suggests that, like we previously observed in spr-5; met-2 mutants, met-2; spr-1 mutants ectopically express MES4 germline genes in somatic tissues. In addition, the fact that the MES-4 germline genes that are affected by loss of SPR- 1 are the same as those affected by the loss of SPR- 5 suggests that they function together on the same MES-4 targets. The ectopic expression of MES-4 germline genes in met-2; spr-1 mutants provides further evidence that SPR-1 is functioning in maternal SPR-5 reprogramming. However, the magnitude of the ectopic expression of MES-4 genes in met-2; spr-1 mutants is intermediate between spr-5; met-2 double mutants and spr-1 or met-2 single mutants. Taken together, these data suggest that SPR-5 functions maternally through its interaction with SPR-1, with loss of SPR-1 partially compromising SPR-5 reprogramming. Consistent with this conclusion, SPR-1 and SPR-5 have been shown to directly interact with one another in C. elegans (Eimer et al., 2002; Kim et al., 2018), and both were identified in a screen for suppressors of presenilin (Wen et al., 2000).

In mammals, epigenetic reprogramming at fertilization also requires LSD1/SPR-5 and SETDB1/MET-2. To determine whether LSD1 reprogramming in mammals requires 
CoREST, we addressed the maternal role of CoREST in mice. We found that CoREST and LSD1 are both expressed during all stages of mouse oocyte development, indicating that both proteins are spatially and temporally positioned for LSD1 to be functioning through CoREST in maternal reprogramming. This is consistent with previous literature describing the expression of LSD1 (Ancelin et al., 2016; Kim et al., 2015b; Wasson et al., 2016) and CoREST (Ma et al., 2012) in the mouse oocyte. Previously, we found that decreased levels of LSD1 protein in the oocyte results in $\sim 30 \%$ perinatal lethality in progeny derived from these mothers (Wasson et al., 2016). Here we show that a M448V mutation, that reduces the ability to bind CoREST, phenocopies the perinatal lethality phenotype observed when LSD1 maternal protein is partially decreased, including the observation that many times all of the animals in a particular litter die. This suggests that the partial requirement for CoREST in maternal LSD1 reprogramming is conserved in mammals. In addition, we detect an allelic series in which the percentage of perinatal lethality increases from $\mathrm{Lsd1}{ }^{+}$to $L s d 1^{\text {het }}$ progeny, and increases again from $L s d 1^{\text {het }}$ to $L s d 1^{M 448 V}$ progeny. The finding that the extent of perinatal lethality is more severe in $L s d 1^{\text {het }}$ progeny compared to $L s d 1^{+}$progeny, provides further evidence that the $L s d 1^{M 448 V}$ allele only partially compromises maternal LSD1 activity. In addition, we find that further compromising maternal LSD1 reprogramming in $L s d 1^{M 448 V}$ progeny compared to $L s d 1^{\text {het }}$ progeny leads to a further increase in perinatal lethality. This strengthens the link that we previously observed (Wasson et al., 2016) between maternal epigenetic reprogramming and defects that manifest postnatally. However, it remains to be determined whether these defects are due to the direct inheritance of inappropriate histone methylation or due to an indirect effect through some other epigenetic mechanism. 


\section{Evidence from diverse developmental processes across multiple phyla support a role}

\section{for CoREST in LSD1 function}

Across multiple phyla, the function of CoREST extends beyond the female germline. In Drosophila, loss of Lsd1 results in sterility in both males and females (Reuter et al. 2007, Di Stefano et al. 2007, Szabad et al. 1988). However, CoRest and Lsd1 also function in the germline support cells. Lsd1 is required in escort cells that support early female germline differentiation (Eliazer et al., 2011), and knockdown of either Lsd1 or CoRest protein causes a number of phenotypes in ovarian follicle cells ( Domanitskaya and Schüpbach, 2012; Lee and Spradling, 2014). The requirement for CoRest in cells that support oogenesis causes sterility in female CoRest mutants. Together, these data potentially implicate CoRest in regulating Lsd 1 function, but the direct role of CoRest has yet to be determined during oogenesis. Furthermore, knockdown of CoRest in Drosophila males phenocopies the male infertility observed in LSD1 knockdown testes (Mačinković et al., 2019). The overlap in phenotypes between Lsd1 and CoRest mutants in the Drosophila spermatogenesis provides further evidence that CoRest may function with LSD1, but it is unclear if that function is in the germline, germline support cells, or both.

Analogous to the partial role for CoREST in C. elegans and mouse LSD1 maternal reprogramming, LSD1 may also be partially dependent on CoREST during mouse embryonic development. The phenotype of homozygous deletion of the Lsd1 gene in mice is lethality by embryonic day 7.5 (e7.5) (Wang et al., 2007; Wang et al., 2009), while the CoREST deleted mice die by e16.5 (Yao et al., 2014). It is possible that the later embryonic 
lethality caused by loss of CoREST could result from the partial loss of LSD1 function, but this remains to be determined.

\section{Potential roles for CoREST in regulating LSD1 activity}

There are two main possibilities for how CoREST may partially regulate LSD1 during maternal reprogramming in C. elegans and mice. One possibility is that CoREST is required for LSD1 activity at a subset of LSD1 targets. For example, it is possible that LSD1 needs CoREST to gain access to chromatin at certain targets that normally exist in more repressed chromatin. Consistent with this possibility, in vitro biochemical experiments showed that while LSD1 can demethylate H3K4 peptides or bulk histones, it is only capable of demethylating nucleosomes when in complex with CoREST (Lee et al., 2005; Shi et al., 2005; Yang et al., 2006). If CoREST is required for helping LSD1 gain access to certain chromatin targets, we would expect that the gene expression changes at these targets would also be completely affected by loss of CoREST. In contrast, at other genes where LSD1 does not need CoREST to gain access to chromatin, the loss of CoREST would not have the same effect as losing LSD1. However, we observe that most genes affected by the loss of LSD1 are also affected by the loss of CoREST, when sensitized by the loss of met-2.

Furthermore, the gene expression changes caused by loss of CoREST are less affected than when LSD1 is lost. This is consistent with an alternative possibility, that CoREST helps LSD1 more efficiently access chromatin genome-wide. In this case, it is possible that SPR-5 maintains sufficient demethylase activity to prevent accumulation of H3K4me1/2 methylation in the absence of SPR-1. For this reason, mutation of SPR-1 alone would not result in a germline mortality phenotype. However, when MET-2 maternal reprogramming 
is lost, the inability to reprogram active chromatin states with $\mathrm{H} 3 \mathrm{~K} 9 \mathrm{me} 1 / 2$ creates a chromatin environment where optimal SPR-5 activity is required. Without SPR-1, the reduced activity of SPR-5 is not sufficient to prevent the germline mortality phenotype that arises in met-2; spr-1 double mutants. Thus, our data are more consistent with a model where CoREST is required maternally to help LSD1 more efficiently access chromatin genome-wide.

\section{Potential implications for CoREST function in humans}

Taken together, our data in C. elegans and mice suggest that CoREST has a conserved role in maternal LSD1 reprogramming. The partial requirement for COREST in LSD1 function has potential implications for putative patients with mutations in COREST. The first human patients with de novo mutations in LSD1 have been identified. These patients display phenotypes that are similar to Kabuki Syndrome, which is characterized by developmental delay and craniofacial abnormalities (Chong et al., 2016; Tunovic et al., 2014). The Lsd1 human mutations appear to be dominant partial loss of function mutations. It is possible that only partial loss of function mutants are viable because of the requirement for LSD1 in embryonic development and in stem cell populations (Haines et al., 2018; Kerenyi et al., 2013; Lambrot et al., 2015; Myrick et al., 2017; Tosic et al., 2018; Zhu et al., 2014). However, if CoREST is also required to help LSD1 more efficiently access chromatin genome-wide in humans, either maternally or zygotically, we might expect that loss of CoREST would readily give rise to similar developmental defects as those caused by partial loss of LSD1 function. As a result, we are actively searching for such potential human COREST patients. 


\section{ACKNOWLEDGEMENTS}

We are grateful to members of the Katz lab, as well as T. Caspary, T. Lee, and C. Bean, for their helpful discussion and critical reading of the manuscript; and the Caenorhabditis Genetics Center (funded by NIH P40 OD010440) for strains. Funding: This work was funded by a grant to D.J.K. (NSF IOS1931697); B.S.C. was supported by the Fellowships in Research and Science Teaching IRACDA postdoctoral program (NIH K12GM00680-15) and by NIH F32 GM126734-01. A.S. was supported by NIH F31 5F31HD098816-03 and the GMB training grant (T32GM008490-21). D.A.M. was supported by a research supplement to promote diversity in health-related research from NINDS (1R01NS087142). This study was supported in part by the Mouse Transgenic and Gene Targeting Core (TMF), which is subsidized by the Emory University School of Medicine and is one of the Emory Integrated Core Facilities. Additional support was provided by the National Center for Advancing Translational Science of the National Institutes of Health (UL1TR000454). Author Contributions: B.S.C., A.S. and D.J.K. conceived and designed the study and wrote the manuscript. B.S.C., A.S., R.G., S.R.C, M.C, and K.S. performed experiments under the direction of D.J.K. B.S.C, A.S., and D.J.K. analyzed data and interpreted results. D.A.M. helped with RNAseq analysis and visualizations. All authors discussed the results. Data availability: Raw and processed genomic data has been deposited with the Gene Expression Omnibus (www.ncbi.nlm.nih.gov/geo) under accession code GSE168081.

\section{MATERIALS AND METHODS}

C. elegans Strains. All Caenorhabditis elegans strains were grown and maintained at $20^{\circ} \mathrm{C}$ under standard conditions, as previously described (Brenner, 1974). The C. elegans spr-5 (by101)(I) strain was provided by R. Baumeister. The N2 Bristol wild-type (WT), spr-1 (ar200)(V), and et1(III) ; et1 [umnls 8 (myo-2p :: GFP + NeoR, III: 9421936)](V) strain was provided by the Caenorhabditis Genetics Center. The met-2 (n4256)(III) strain was provided by R. Horvitz. From these strains we generated spr-1 (ar200) (V)/ et1 [umnls 8 (myo-2p :: GFP + NeoR, III: 9421936)](V) and met-2 (n4256) (III) / et1 [umnls 8 (myo-2p :: GFP + NeoR, III: 9421936)](V); spr-1 (ar200)(V)/ et1 [umnls 8 (myo-2p :: GFP + NeoR, III: 9421936)](V). For genotyping, single animals were picked into $5-10 \mathrm{ul}$ of lysis buffer $(50 \mathrm{mM} \mathrm{KCl}, 10 \mathrm{mM}$ Tris- $\mathrm{HCl}$ (pH 8.3), 2.5mM MgCl2, 0.45\% NP-40, 0.45\% Tween-20, 0.01\% gelatin) and 
incubated at $65^{\circ} \mathrm{C}$ for 1 hour followed by $95^{\circ} \mathrm{C}$ for 30 minutes. PCR reactions were performed with AmpliTaq Gold (Invitrogen) according to the manufacturer's protocol and reactions were resolved on agarose gels.

Generation of M448V hypomorphic allele. Oligos were designed to include an A>G SNP conversion which removed an HpyAV restriction site, and a G>A PAM blocking silent SNP. C57BL/6 females were superovulated by injecting $0.1 \mathrm{~mL} /$ head of CARD HyperOva (i.p.) on day 1. After 48 hours, females were injected with 7.5IU human chorionic gonadotropin (hCG, i.p.). Oocytes were collected 13 hours after the administration of hCG and fertilized with C57BL/6 sperm in vitro. Five hours postfertilization, 50ng/uL Cas9mRNA, 50ng/uL oligo, and 50ng/uL sgRNA were injected into the cytoplasm of embryos. Injected embryos were incubated at $37^{\circ} \mathrm{C}$ overnight. Two-cell embryos were then transferred into the oviducts of pseudopregnant females. Progeny of those females were genotyped for the point mutation, mated, and progeny were genotyped again to ensure the mutation passed through the germline. Mutant animals were backcrossed at least s two times to C57BL/6 animals before being used in experiments.

Mouse husbandry and genotyping. The following mouse strains were used: $\mathrm{Zp3}$-Cre MGI:2176187 (de Vries et al. 2000), Lsd1fl/fl MGI: 3711205 (Wang et al. 2007), C57BL/6 MGI: 3715241, and Lsd1 $1^{M 448 V}$. Primers for Lsd1 forward (F): GCACCAACACTAAAGAGTATCC, Lsd1 reverse (R): CCACAGAACTTCAAATTACTAAT. A wild type allele of Lsd1 results in a 720 base pair (bp) product, the floxed allele is $480 \mathrm{bp}$, and the deleted allele is $280 \mathrm{bp}$. Primers for Cre F: GAACCTGATGGACATGTTCAGG, Cre R: 
Cre ctrl R: TGGGCTGGGTGTTAGCCTTA. If Cre+, this results in a 302 bp product, and Cre ctrl F/R primers are an internal control that yields a 250bp product. Primers for M448V F: CCCAAATGGCATGACATAAA, M448V R: TAAGGCACCAAACCCCTTCT resulting in a 386bp product. The point mutation removes a restriction site, so mutants versus wild type were determined by incubating PCR products at $37^{\circ} \mathrm{C}$ with the HpyAV restriction enzyme for one hour. Wild type band sizes: 72bp, 81bp, 209bp, 24bp. M448V band sizes: 72bp, 290bp, 24bp. All mouse work was performed under protocols approved by the Emory University Institutional Animal Care and Use Committee.

Immunofluorescence. Mice were sacrificed by cervical dislocation and ovaries were isolated. Ovaries were then fixed in 4\% PFA for one hour, followed by four PBS washes over two hours. Tissues were cryoprotected in $30 \%$ sucrose at $4^{\circ} \mathrm{C}$ overnight and then embedded in O.C.T. Compound (Tissue Tek). Cryosections were obtained at $10 \mu \mathrm{m}$ and immunostaining was performed using rabbit polyclonal anti-LSD1 (1:200, ab17721), rabbit polyclonal anti-CoREST (1:100, LS-B8140-50), and Alexa fluor conjugated secondary antibodies (1:500).

Perinatal lethality. Breeding cages were observed daily for new litters and number born alive were scored at P0. At P1, litter sizes were scored again, and percent lethality was calculated by determining the number of animals that died divided by the original size of the litter. Those that died due to failure to thrive shortly after birth were often missing visible milk spots. Only litters from mothers $<8$ months of age were used to avoid complications due to advanced maternal age.

Germline mortality assay. The germline mortality experiments were performed as described by Katz and colleagues (Katz et al., 2009). In brief, worms were maintained at 
$20^{\circ} \mathrm{C}$ and three fertile young adults with visible embryos were transferred to new NGM plates every four days. The total number of progeny from wild type, spr-1 mutants, and spr5 mutants was counted every third generation until generation 17, after which counts were completed every other generation. Average number of progeny from spr-5 mutants was calculated from 10 animals until counts were stopped at generation 41 due to the inability to maintain fertile animals. For wild type, the average number of progeny was calculated from five animals until generation 41 when the average number of progeny was calculated from six animals. The average number of progeny from spr-1 mutants was calculated from ten animals throughout the entirety of the experiment. The same germline morality assay was adapted to evaluate the germline mortality of wild-type, spr-1 and met-2 single mutants, and met-2; spr-1 double mutants. Here, the number of progeny were counted every generation, except from spr-1 mutants which was counted every fourth generation. The average number of progeny from wild type, spr-1 mutants and met-2 mutants was calculated from ten animals, while met-2; spr-1 was calculated from 30 animals. The standard error of the mean (SEM) was calculated for each generation the number of progeny were averaged.

RNA sequencing and analysis. Total RNA was isolated using TRIzol reagent (Invitrogen) from $\sim 500-1000$ starved L1 larvae hatched at room temperature $\left(21^{\circ} \mathrm{C}-22^{\circ} \mathrm{C}\right)$ overnight in M9 Buffer. L1 larvae from wild type, spr-1, met-2, and met-2; spr-1 were isolated at generation 7 (F7) prior to the observed decrease in sterility. For each genotype, 2 biological replicates were obtained. Sequencing reads were checked for quality using FastQC (Wingett and Andrews, 2018), filtered using Trimmomatic (Bolger et al., 2014), and remapped to the $C$. elegans transcriptome (ce10, WS220) using HISAT2 (Kim et al., 2015a). 
Read count by gene was obtained by FeatureCounts (Liao et al., 2014). Differentially expressed transcripts (significance threshold, Wald test, $\mathrm{p}$-value $<0.05$ ) were determined using DESEQ2 (v.2.11.40.2) (Love et al., 2014). Transcripts per million (TPM) values were calculated from raw data obtained from FeatureCounts output. Subsequent downstream analysis was performed using $\mathrm{R}$ with normalized counts and p-values from DESEQ2 (v.2.11.40.2). Heatmaps were produced using the ComplexHeatmap R Package (Gu et al., 2016). Data was scaled and hierarchical clustering was performed using the complete linkage algorithm. In the linkage algorithm, distance was measured by calculating pairwise distance. Volcano plots were produced using the EnhancedVolcano package (v.0.99.16). Additionally, Gene Ontology (GO) Pathway analysis was performed using the online platform WormEnrichr (Chen et al., 2013; Kuleshov et al., 2016). An additional heatmap comparison of differentially expressed genes between spr-1, met-2, met-2; spr-1 and spr-5; met-2 progeny compared to wild type progeny was generated in Microsoft Excel using log2 fold change values from the DESEQ2 analysis. Differentially expressed genes in spr-5; met2 double mutants compared to wild type examined in this manuscript were obtained from a separate RNAseq analysis performed under the same conditions (Carpenter et al., 2021). Because transcript isoforms were ignored, we discuss the data in terms of "genes expressed" rather than "transcripts expressed".

Differential interference contrast (DIC) microscopy. Worms were immobilized in $0.1 \%$ levamisole and placed on a $2 \%$ agarose pad for imaging at either $10 \mathrm{x}, 40 \mathrm{x}$, or $100 \mathrm{x}$ magnification. 


\section{Figures}

A
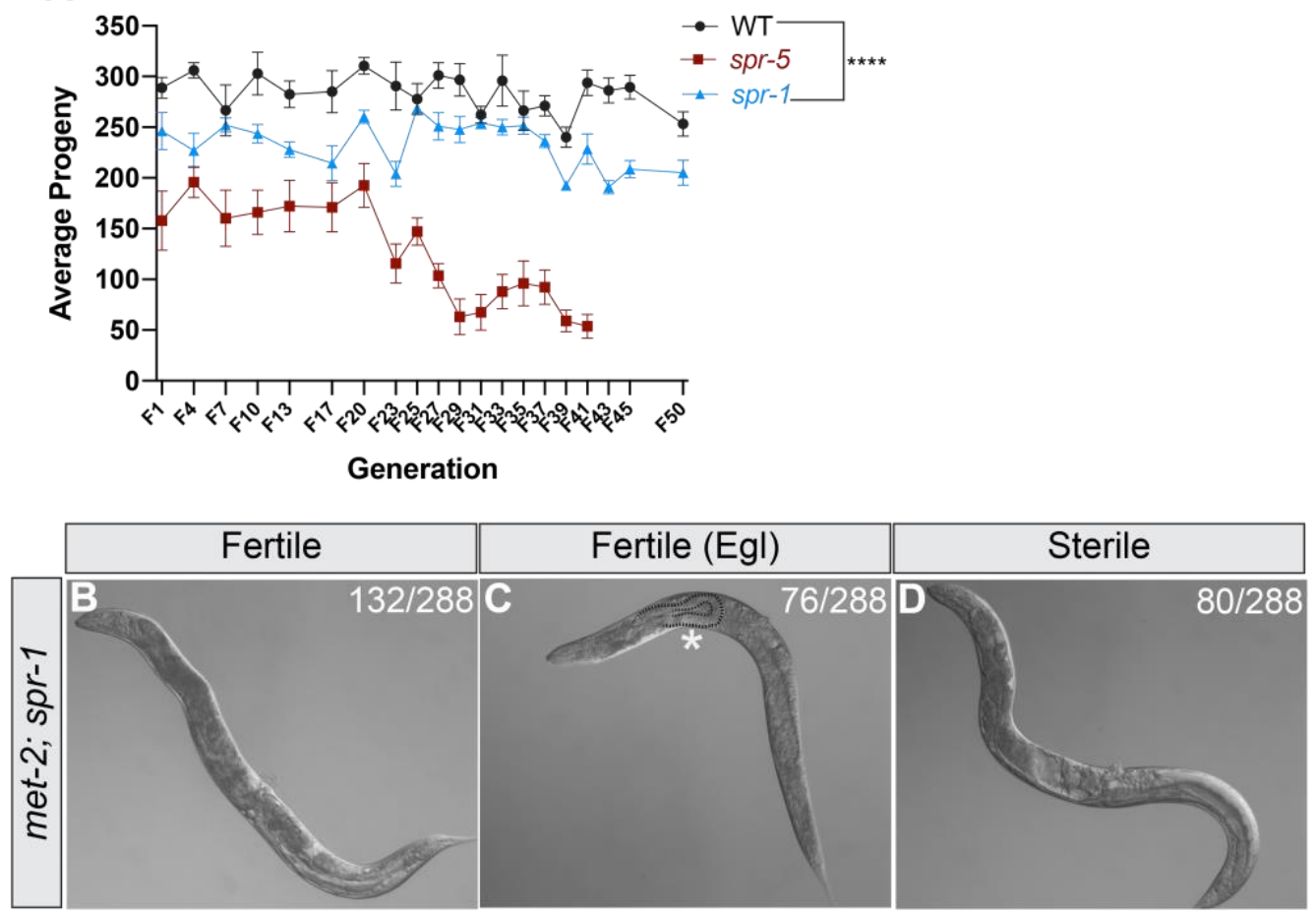

E
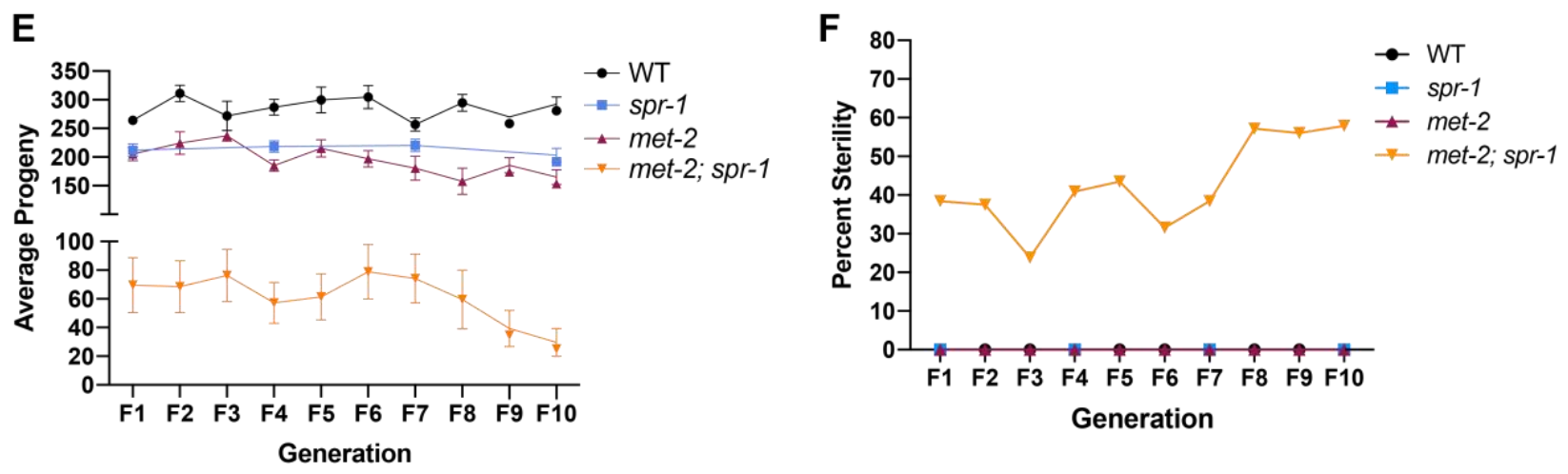

Figure 1. Germline mortality in spr-1 and met-2; spr-1 mutants. (A) The average number of total progeny from wild type (WT), spr-5, and spr-1 mutants over progressive generations. The average number of progeny from $s p r-1$ mutants $(\mathrm{N}=192, \mathrm{~N}=$ total number broods counted) was significantly decreased compared to WT animals (N=92) across 50 generations (unpaired student t-test, ${ }^{* * * *}$ p-value <0.0001). 10x DIC images of first generation (F1) met-2; spr-1 mutants scored as either fertile (B), fertile (Egl) (C), or sterile (D). Asterisk denotes hatched larvae outlined by a dashed line inside of a met-2; spr-1 mutant scored as fertile (Egl)(C). (E) The average number of total progeny from WT, spr-1, met-2 and met-2; spr-1 mutants over progressive generations. Error bars in (A, E) represent the standard error of the mean (SEM). (F) Percent of animals cloned out for experiment in (E) scored for sterility over progressive generations. spr-1 mutant progeny were only scored at F1, F4, F7, and F10 generations in (E, F). 


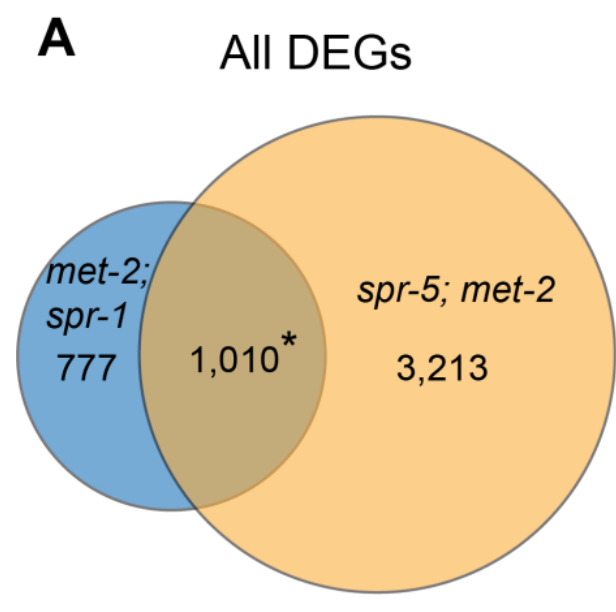

B Up-regulated DEGS

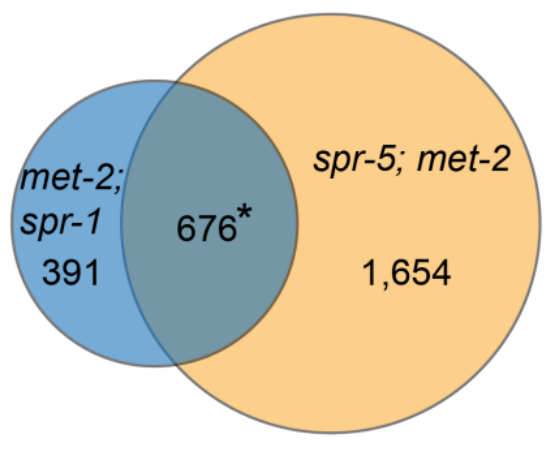

$\mathbf{E}$ 676 overlapping DEGs

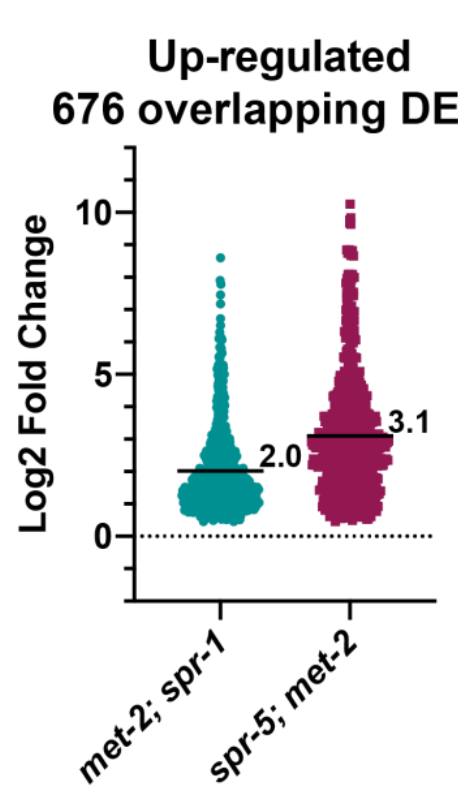

D
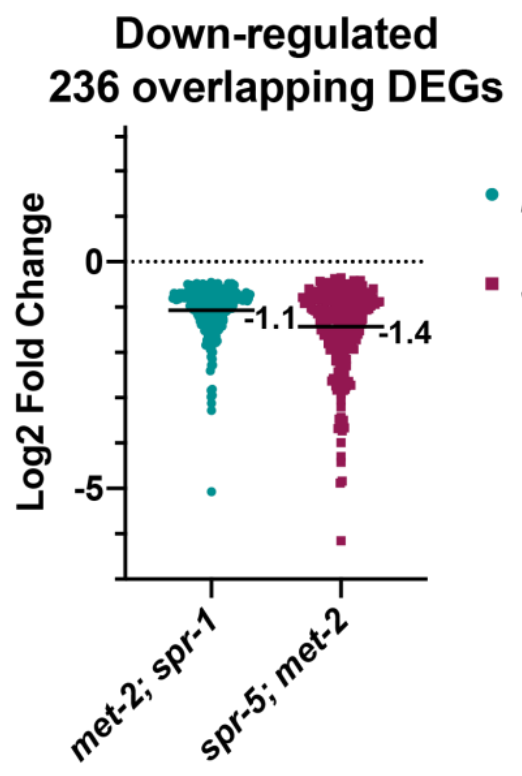

C Down-regulated DEGS

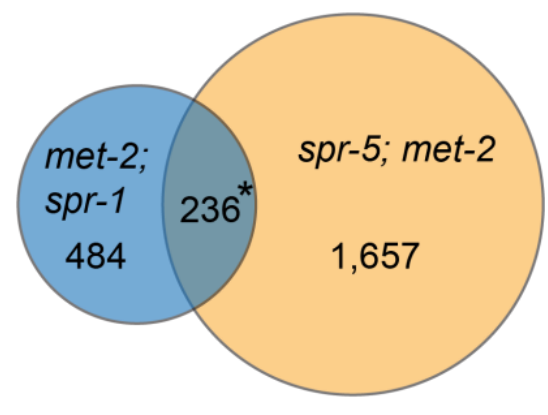

- met-2; spr-1

- spr-5; met-2

Figure 2. Transcriptional misregulation in met-2; spr-1 progeny resembles that observed in spr-5; met-2 progeny but is less affected. Overlap between all (A), upregulated (B), and down-regulated (C) differentially expressed genes (DEGs) in met-2; spr-1 and spr-5; met-2 L1 progeny. Significant over-enrichment in A-C was determined by the hypergeometric test $\left({ }^{*} \mathrm{P}\right.$-value $<1.28 \mathrm{E}-270$, ${ }^{*} \mathrm{P}$-value $<2.61 \mathrm{E}-392,{ }^{*} \mathrm{P}$-value $<2.16 \mathrm{E}-72$, respectively). Scatter dot plots displaying the log2 fold change of the 676 up-regulated (D), and 236 down-regulated (E) overlapping DEGs between met-2; spr-1 and spr-5; met-2 progeny. (D, E) Numbers and solid black lines represent the mean log2 fold change. DEGs in spr-5; met-2 progeny were obtained from (Carpenter et al., 2021). 
A

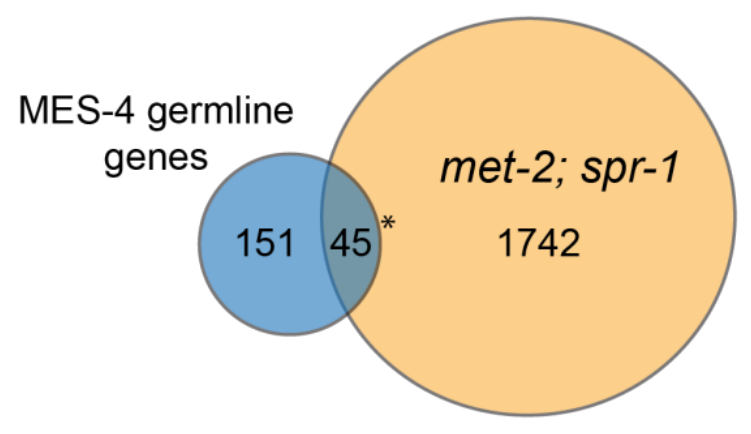

B MES-4 germline genes met-2; spr-1

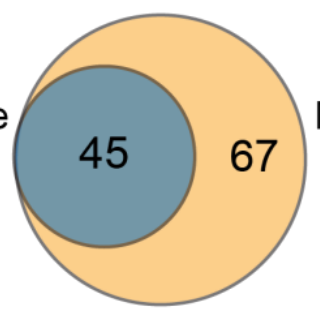
spr-5; met-2 MES-4 germline genes

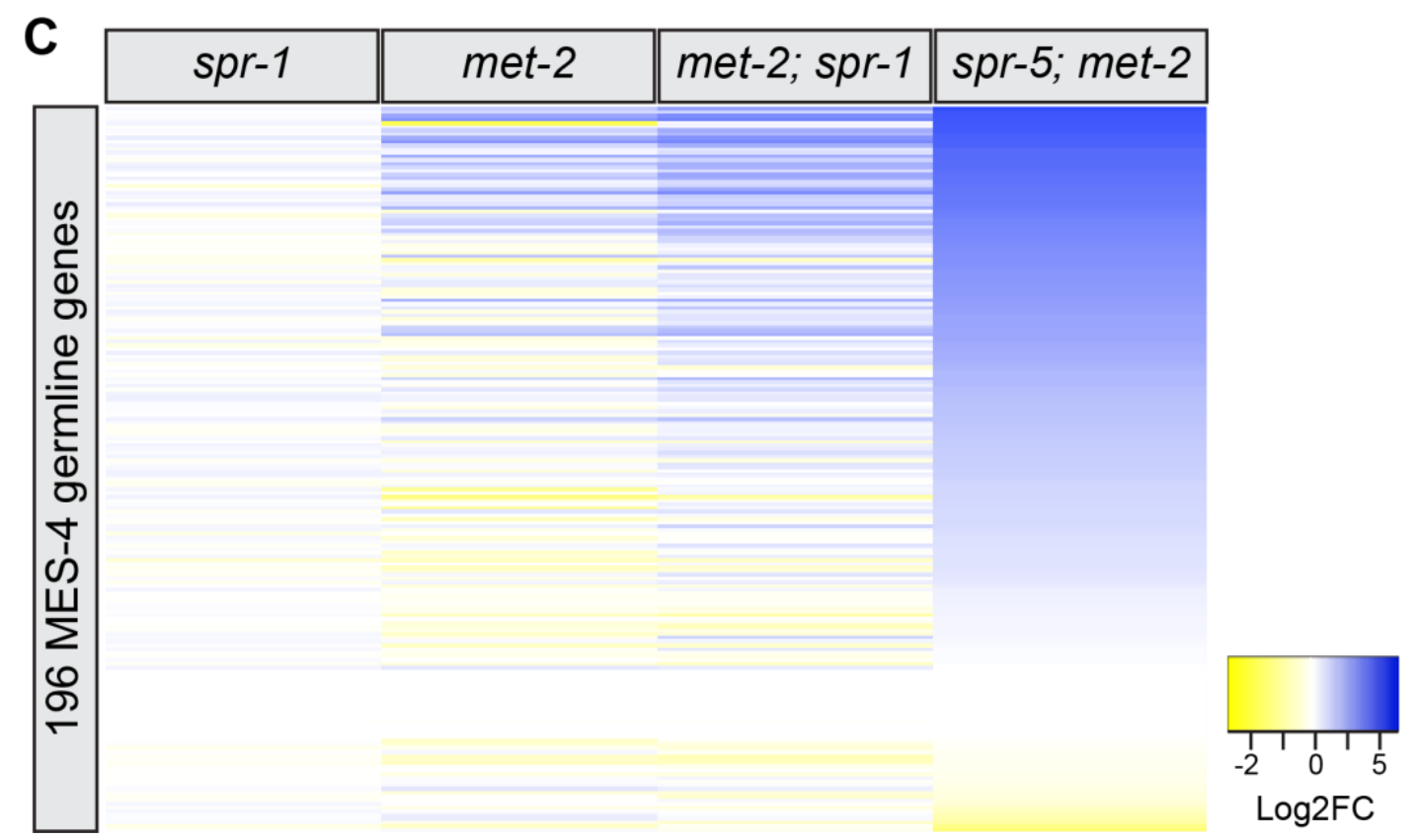

Figure 3. MES-4 germline genes are enriched in met-2; spr-1 mutants, but less affected compared to spr-5; met-2 mutants. (A) Overlap between MES-4 germline genes and differentially expressed genes (DEGs) in met-2; spr-1 L1 progeny. Asterisks denotes significant over-enrichment in A as determined by a hypergeometric test (P-value $<1.41 \mathrm{E}$ 9). (B) Overlap between MES-4 germline genes differentially expressed in met-2; spr-1 and spr-5; met-2 L1 progeny. (C) Heatmap of log2 fold change (FC) of all 196 MES-4 germline genes in spr-1, met-2, met-2; spr-1 and spr-5; met-2 mutants compared to wild type. $\log 2(\mathrm{FC})$ values are represented in a yellow to blue gradient with a range of -2 to 5 . Yellow represents genes with negative $\log 2(\mathrm{FC})$ values and blue represents genes with positive $\log 2 \mathrm{FC}$ values compared to wild type. 

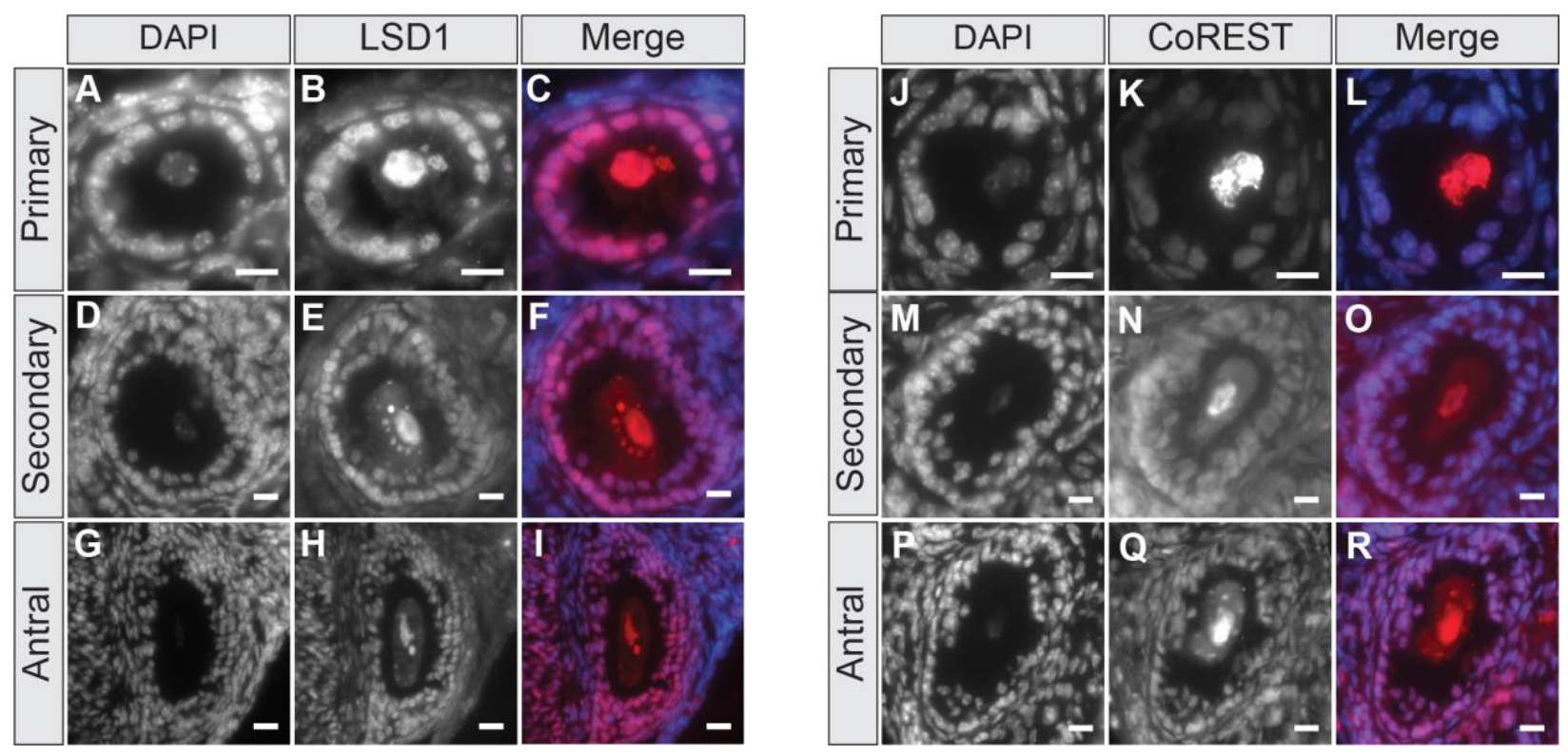

Figure 4. LSD1 and CoREST are expressed during each stage of mouse oocyte development. Representative immunofluorescence images of various stages of the mouse oocyte: primary (A-C, J-L), secondary (D-F, M-O), and antral (G-I, P-R). DAPI (A, D, G, J, M, P), LSD1 (B, E, H) CoREST (K, N, Q), and Merge (C, F, I, L, O, R). Both LSD1 and CoREST are expressed in the oocyte nucleus and surrounding follicle cells during each stage of oocyte development. Scale bars $=25 \mathrm{um}$. 
A

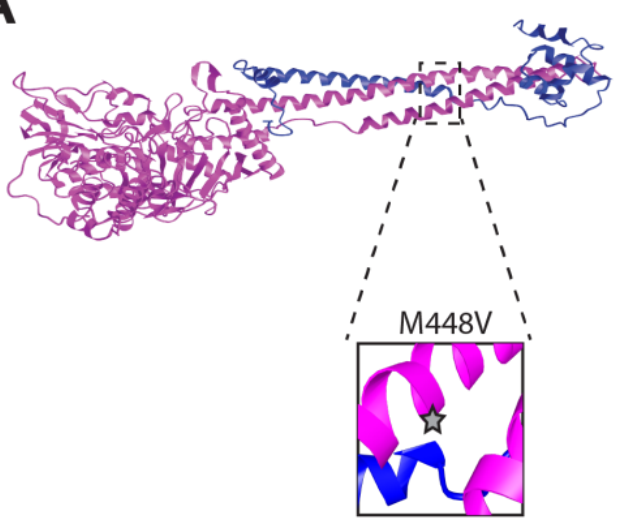

C

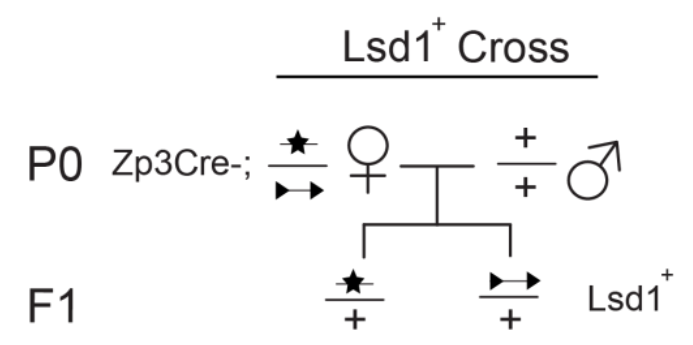

B

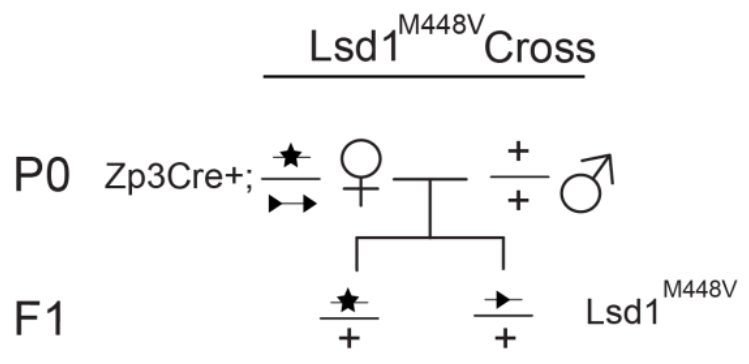

D

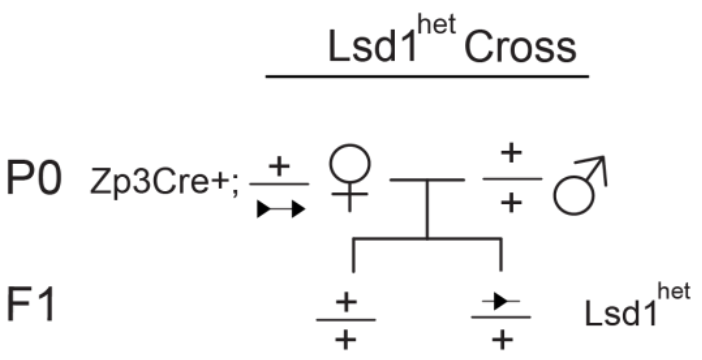

E

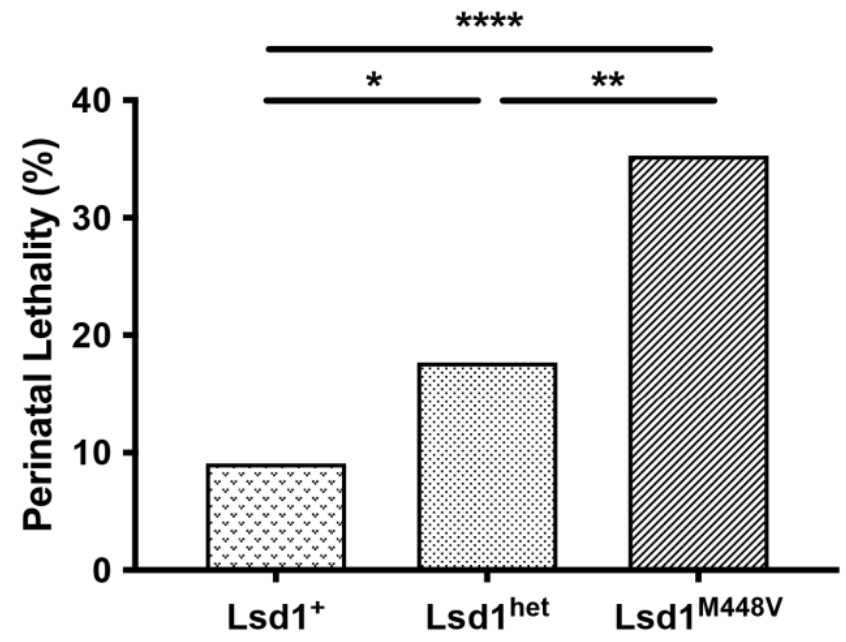


Figure 5. Hypomorphic maternal LSD1 results in perinatal lethality. (A) Crystal structure of LSD1 (pink) in complex with CoREST (blue) from Nicholson et al., 2013. The M448V mutation is in a CoREST binding site (star). (B-D) Genetic crosses showing wild type (+), loxP sites (triangles), and M448V (star) alleles. In all cases, P0 females are crossed to wild-type males, so that F1 progeny have normal zygotic LSD1 activity from their paternal allele after transcription begins at the 2 -cell stage. (B) In the $L s d 1^{M 448 V}$ cross, P0 mothers are Zp3Cre+, contributing only the hypomorphic allele maternally. (C) In the Lsd $1^{+}$ control cross, P0 mothers are Zp3Cre-, contributing a wild-type and hypomorphic allele maternally. (D) In the Lsd1 het control cross, P0 mothers are Zp3Cre+, contributing one wild-type copy of $L s d 1$ maternally. (E) Percent perinatal lethality per litter by experimental condition, $\mathrm{n}=154$ pups from 24 litters (Lsd1 $1^{+}$), $\mathrm{n}=96$ pups from 15 litters (Lsd1 het), and $\mathrm{n}=$ 187 pups from 32 litters (Lsd1 ${ }^{\mathrm{M} 448 \mathrm{~V}}$ ). See Supplemental file 1 for the list of individual litters. $\mathrm{p}$ values calculated using a chi-square test, ${ }^{* * * *}=\mathrm{p}<.0001,{ }^{* *}=\mathrm{p}<.01,{ }^{*}=\mathrm{p}<.05$. 


\section{Supplemental Material}

\section{Supplemental Figure 1}

A

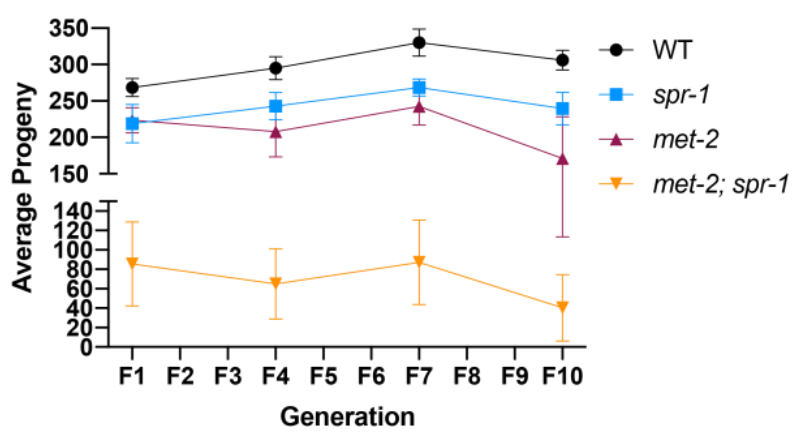

C

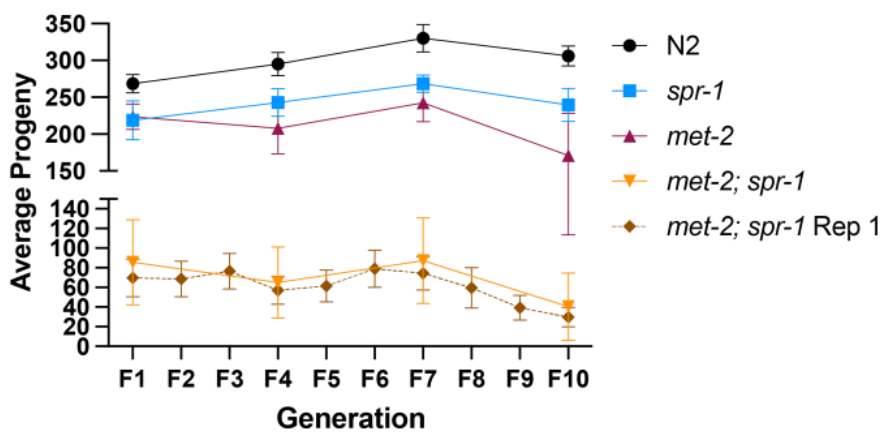

B

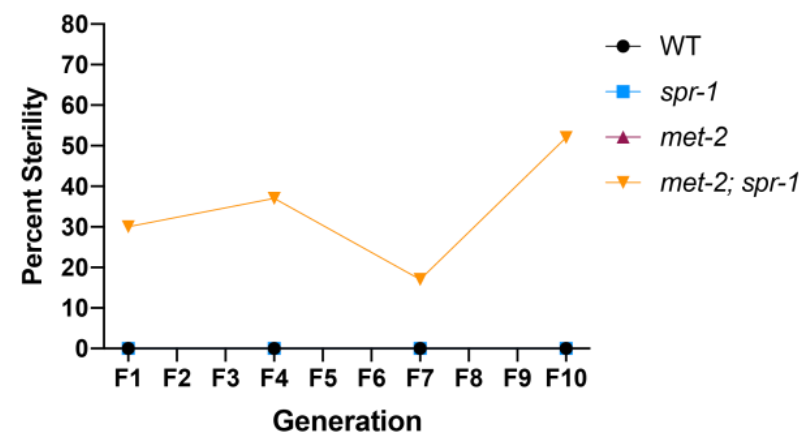

D

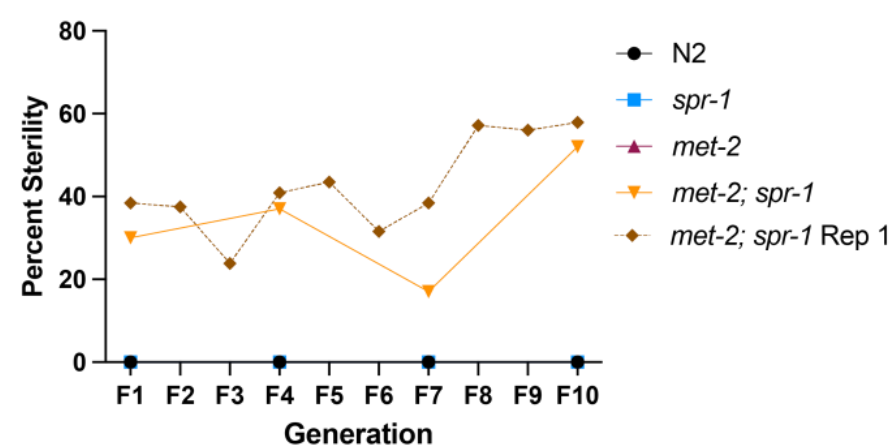

Figure S1. Germline mortality in spr-1 and met-2; spr-1 mutants replicate experiment. (A) The average number of total progeny from wild type (WT), spr-1, met-2 and met-2; spr-1 mutants over progressive generations in a repeat experiment (see Fig. 1E for comparison). (B) Percent of animals cloned out for repeat experiment in (A) scored for sterility over progressive generations (see Fig. 1F for comparison). (C) Same as (A) with overlay of total progeny from met-2; spr-1 (met-2; spr-1 Rep 1) mutants from germline mortality experiment in Figure 1E. (D) Same as (B) with overlay of percent sterile met-2; spr-1(met-2; spr-1 Rep 1) mutants in Figure 1F. Error bars in (A, C) represent the standard error of the mean (SEM). Animals cloned out for the replicate germline mortality experiment were only scored at F1, F4, F7, and F10 generations in (A-D). 


\section{Supplemental Figure 2}

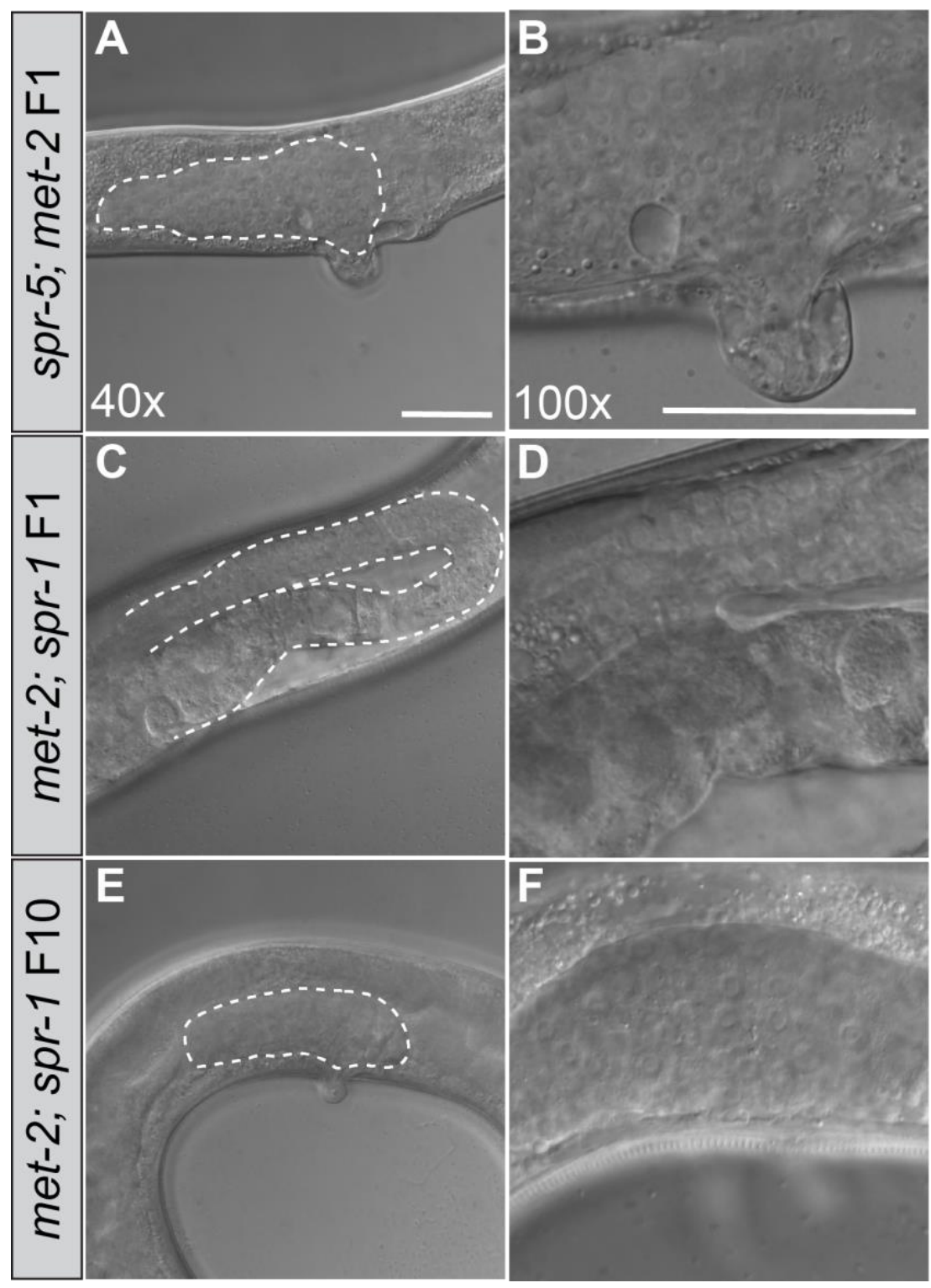

Figure S2. Sterile spr-5; met-2 and met-2; spr-1 double mutant gonads.

40x Differential Interference Contrast (DIC) images of F1 spr-5; met-2 (A) F1 met-2; spr-1 (C), and F10 met-2; spr-1 (E) adult germlines. White dotted lines denote germlines. 100x DIC images of F1 spr-5; met-2 (B) F1 met-2; spr-1 (D), and F10 met-2; spr-1 (F) adult germlines. Scale bar: $100 \mu \mathrm{m}$. 


\section{Supplemental Figure 3}

A

\begin{tabular}{|c|c|c|c|}
\hline Genotype & $\begin{array}{c}\text { Total \# of } \\
\text { differentially } \\
\text { expressed genes }\end{array}$ & Up-regulated & Down-regulated \\
\hline$s p r-1$ & 60 & 41 & 19 \\
\hline met-2 & 1327 & 713 & 614 \\
\hline met-2; spr-1 & 1787 & 1067 & 720 \\
\hline
\end{tabular}

B

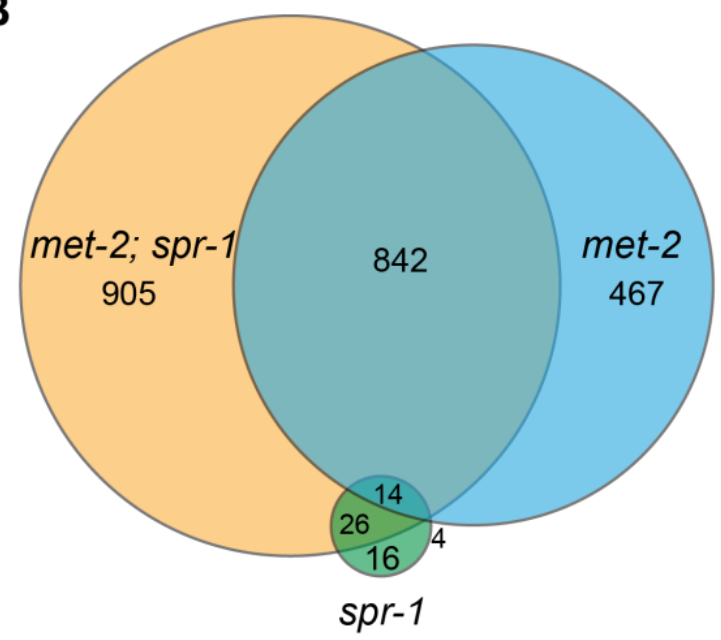

TOP Gene Ontology

GO Biological Processes

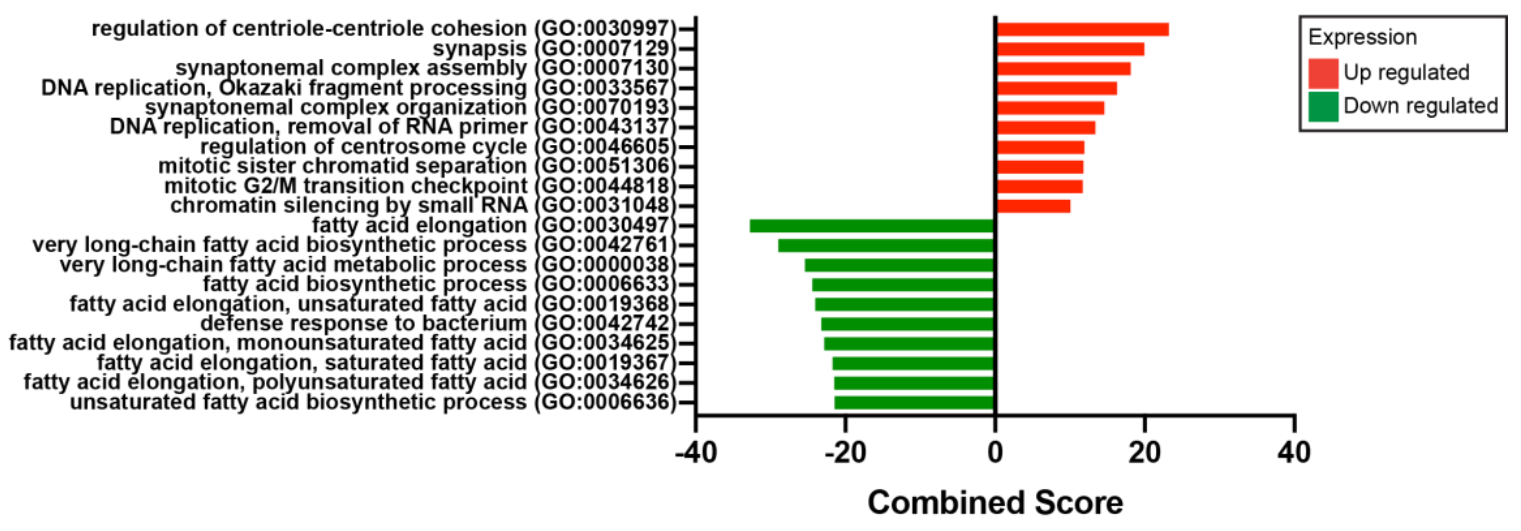

D

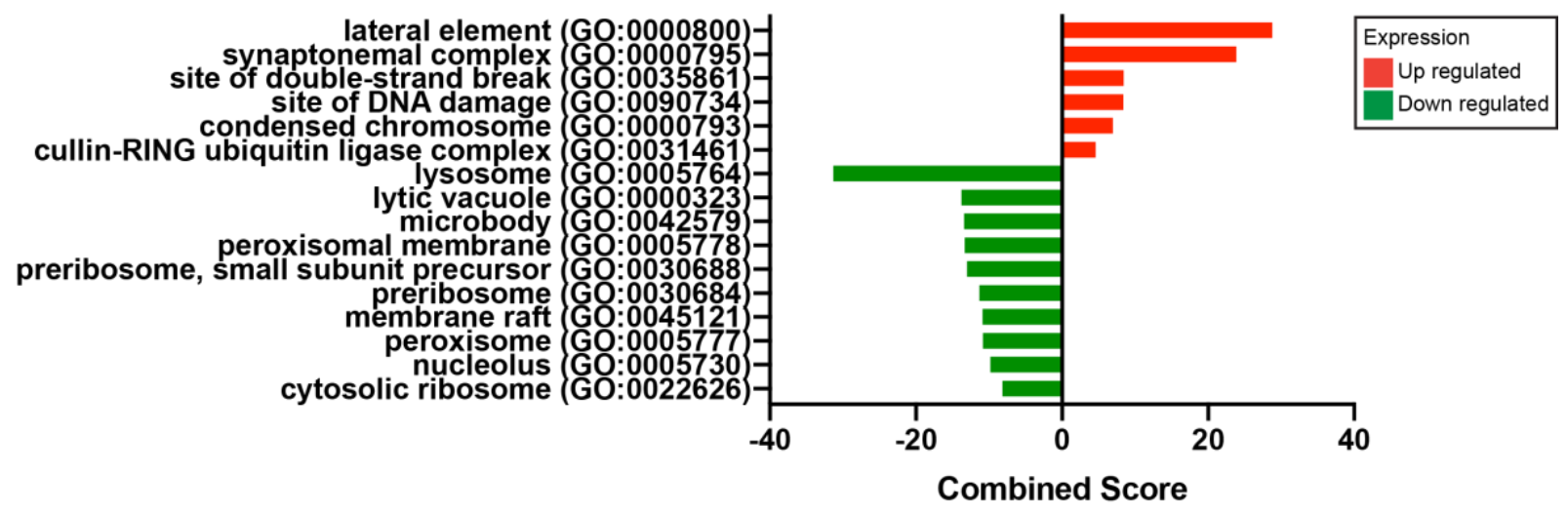


Figure S3: Differential gene expression spr-1, met-2, and met-2; spr-1 progeny compared to wild type. (A) Table summary of differentially expressed genes (DEGs) in spr-1, met-2, and met-2; spr-1 progeny from DeSEQ2 analysis (significance cut-off of p-adj< 0.05). (B) Overlap of differentially expressed genes between spr-1, met-2, and met-2; spr-1 L1 progeny. Gene Ontology analysis showing Biological Processes (C) and Cellular Components (D) amongst genes that were up-regulated (red) and down-regulated (green) in met-2; spr-1 progeny compared to wild-type. Combined Score was computed to determine gene category enrichment (Chen et al., 2013). 


\section{Supplementary Figure 4}
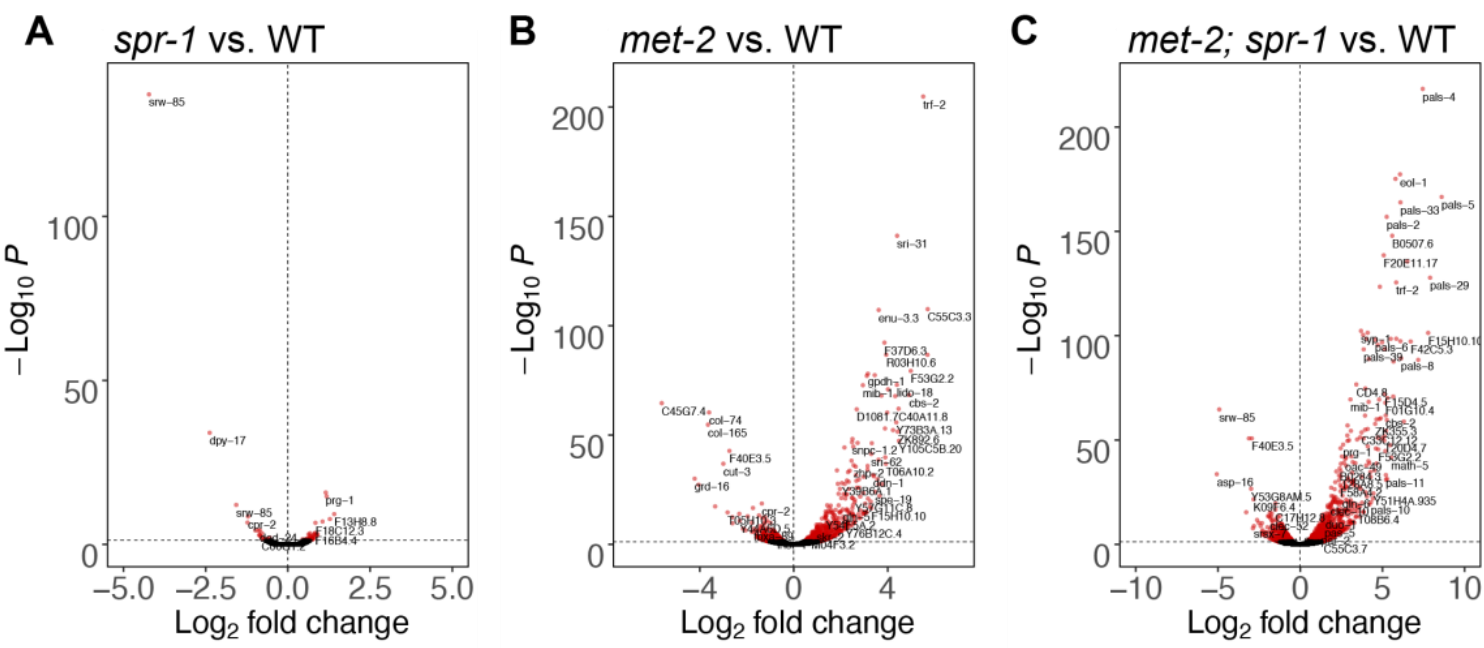

D

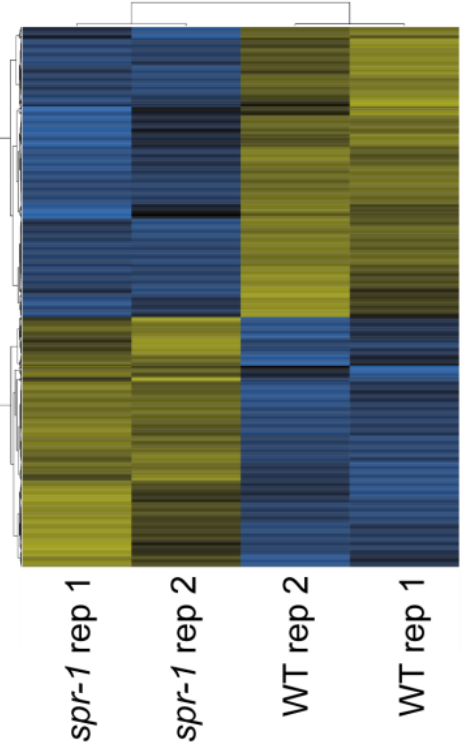

E

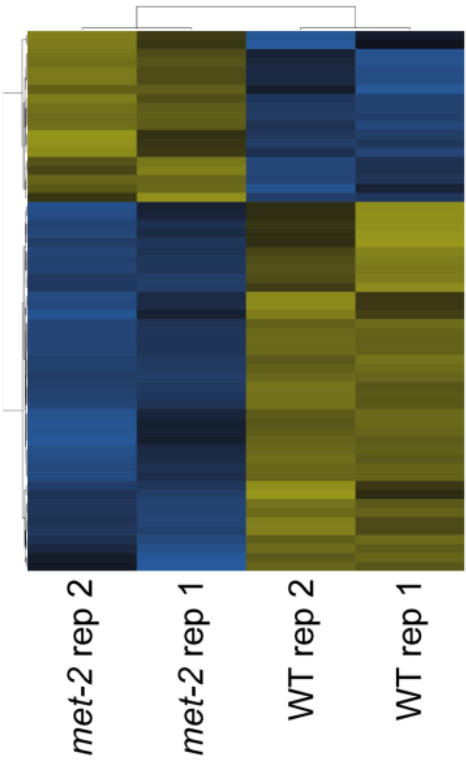

$\mathbf{F}$

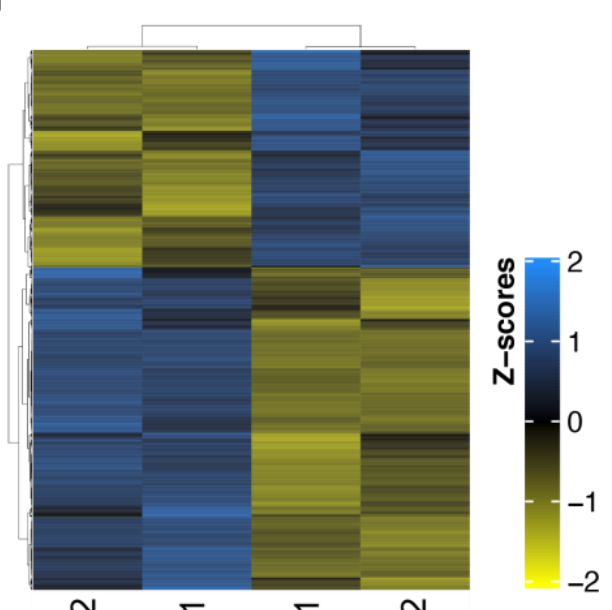

Figure S4: Differential expression and replicate comparison of RNAseq experiments performed on wild type, spr-1, met-2, and met-2; spr-1 progeny. Volcano plot of log2 fold changes in gene expression ( $\mathrm{x}$-axis) by statistical significance (- $\log _{10} \mathrm{P}$-value; $\mathrm{y}$-axis) in spr-1 (A), met-2 (B), and met-2; spr-1 (C) L1 Progeny compared to wild type (WT). Heatmap of differentially expressed RNA-seq transcripts between WT and spr-1 (D), met-2 (E), and met-2; spr-1 (F). Data was scaled and hierarchical clustering was performed using complete linkage algorithm, with distance measured by calculating pairwise distance. Higher (blue) and lower (yellow) expression is reported as a z-score. 


\section{Supplementary Figure 5}

A

Donor

sgRNA

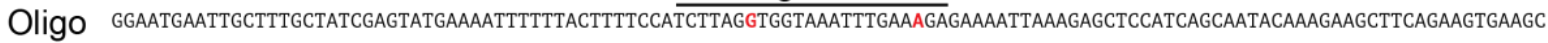
Sequence $\begin{array}{cc}\text { A>G SNP } & \begin{array}{c}\text { G>A PAM } \\ \text { conversion }\end{array} \\ \text { blocking } \\ \text { silent SNP }\end{array}$

B Superovulate female In vitro fertilization Inject fertilized embryo $\quad \begin{gathered}\text { into pseudopregnant } \\ \text { female }\end{gathered}$
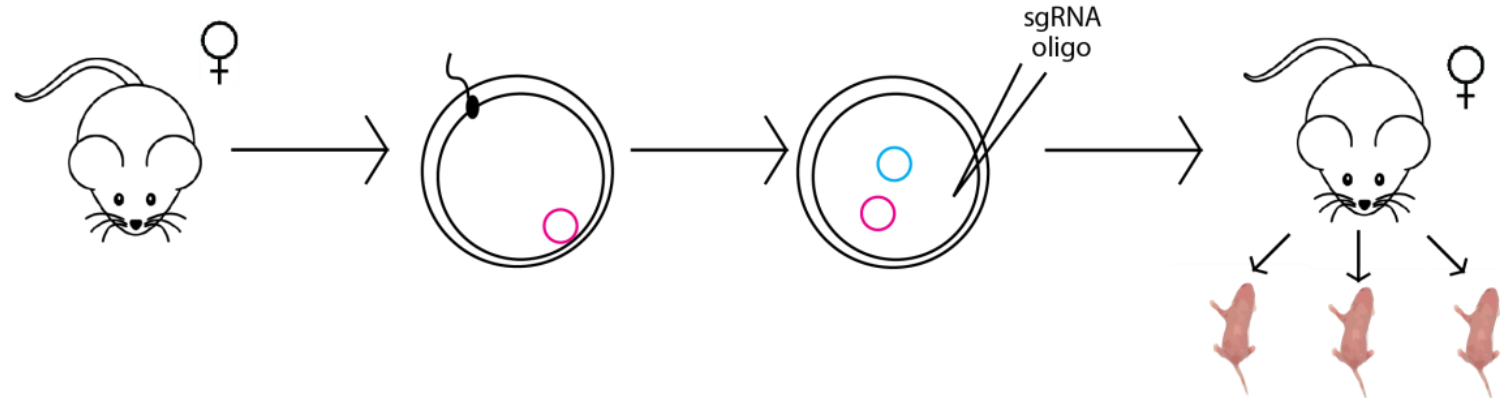

C

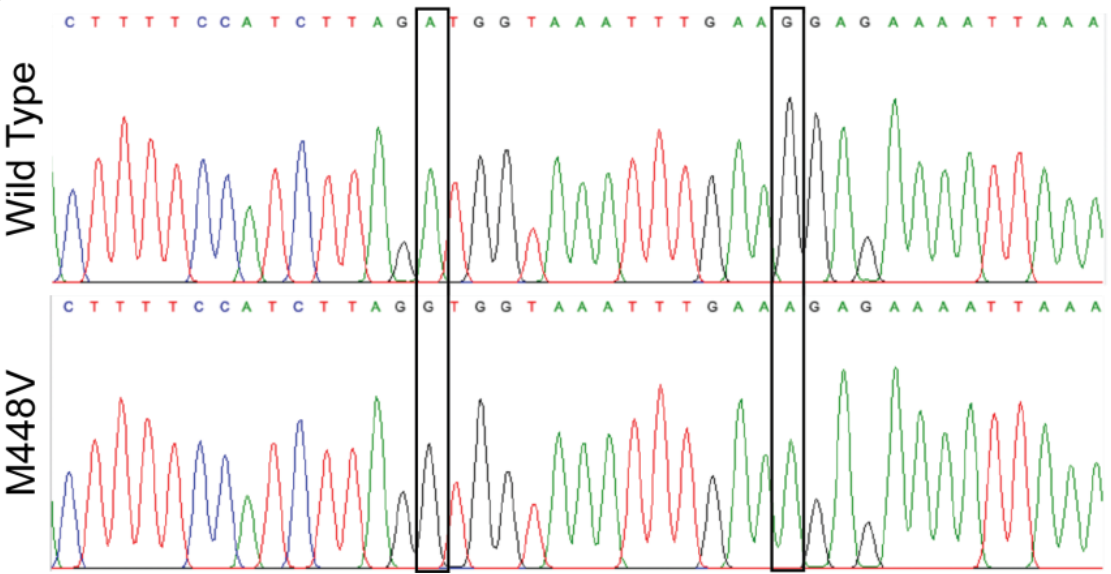

Genotype offspring for M448V

Figure S5. Generation of M448V hypomorphic allele. (A) Donor oligo sequence with the sgRNA sequence denoted. Two point mutations being introduced are colored in red: $A>G$ SNP and G>A PAM blocking silent SNP. (B) Workflow for introducing M448V mutation into mice. (C) Wild-type chromatogram versus validated $A>G$ SNP and G>A silent SNP in the newly generated mutant. 


\section{Supplementary Figure 6}

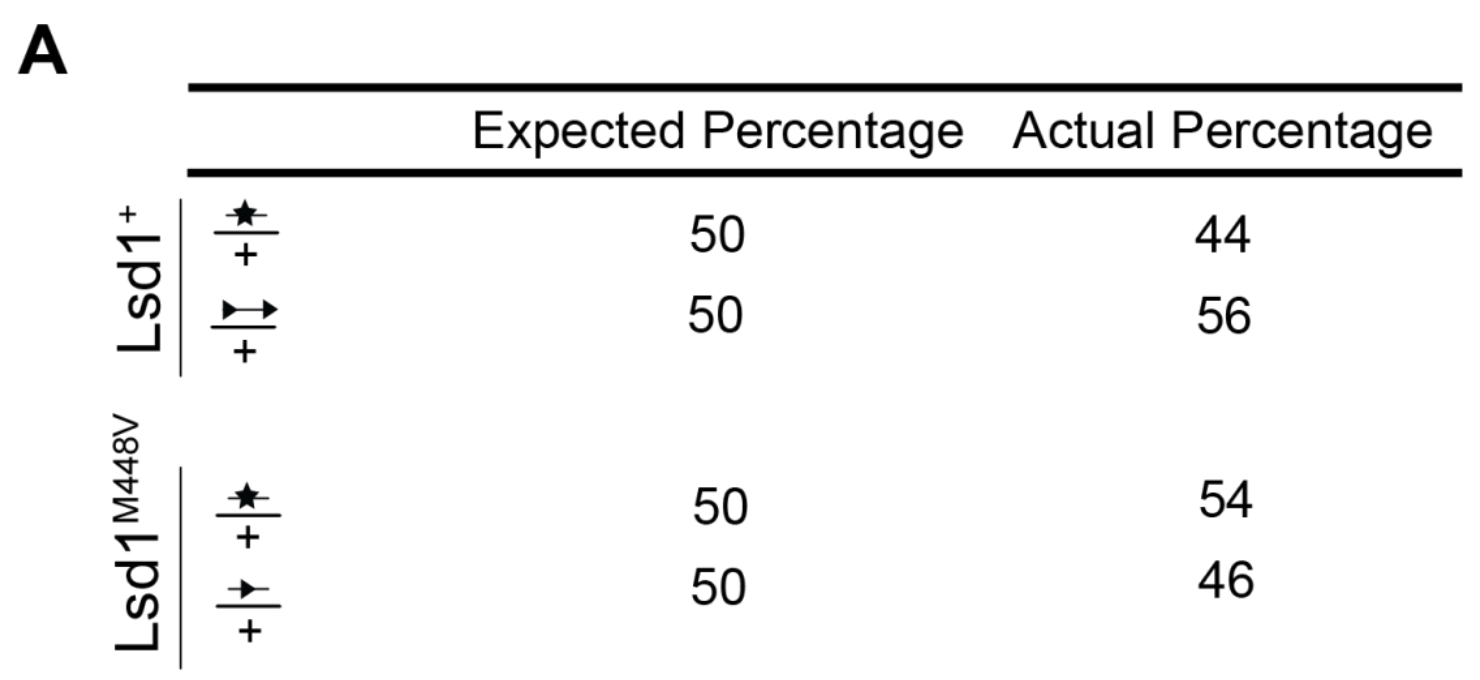

Figure S6. Percent survival by genotype per experimental condition. $L s d 1^{+} \mathrm{n}=50$ animals genotyped. $L s d 1^{M 448 V} \mathrm{n}=87$. Statistical analyses performed using chi square test, no significant difference between expected and actual percentages for either experimental condition.

\section{Supplemental files}

Supplemental file 1. List of individual litters analyzed for embryonic lethality in Figure 5. 


\section{REFERENCES}

Ancelin, K., Syx, L., Borensztein, M., Ranisavljevic, N., Vassilev, I., Briseño-Roa, L., Liu, T., Metzger, E., Servant, N., Barillot, E., et al. (2016). Maternal LSD1/KDM1A is an essential regulator of chromatin and transcription landscapes during zygotic genome activation. Elife 5, 4615.

Bannister, A. J., Schneider, R., Myers, F. A., Thorne, A. W., Crane-Robinson, C. and Kouzarides, T. (2005). Spatial distribution of di- and tri-methyl lysine 36 of histone H3 at active genes. J Biol Chem 280, 17732-17736.

Barski, A., Cuddapah, S., Cui, K., Roh, T.-Y., Schones, D. E., Wang, Z., Wei, G., Chepelev, I. and Zhao, K. (2007). High-resolution profiling of histone methylations in the human genome. Cell 129, 823-837.

Bernstein, B. E., Humphrey, E. L., Erlich, R. L., Schneider, R., Bouman, P., Liu, J. S., Kouzarides, T. and Schreiber, S. L. (2002). Methylation of histone H3 Lys 4 in coding regions of active genes. PNAS 99, 8695-8700.

Bernstein, B. E., Kamal, M., Lindblad-Toh, K., Bekiranov, S., Bailey, D. K., Huebert, D. J., McMahon, S., Karlsson, E. K., Kulbokas, E. J., Gingeras, T. R., et al. (2005). Genomic maps and comparative analysis of histone modifications in human and mouse. Cell 120, 169-181.

Bolger, A. M., Lohse, M. and Usadel, B. (2014). Trimmomatic: a flexible trimmer for Illumina sequence data. Bioinformatics 30, 2114-2120.

Brenner, S. (1974). The genetics of Caenorhabditis elegans. Genetics 77, 71-94.

Brykczynska, U., Hisano, M., Erkek, S., Ramos, L., Oakeley, E. J., Roloff, T. C., Beisel, C., Schübeler, D., Stadler, M. B. and Peters, A. H. F. M. (2010). Repressive and active histone methylation mark distinct promoters in human and mouse spermatozoa. Nat Struct Mol Biol 17, 679-687.

Burton, A. and Torres-Padilla, M.-E. (2014). Chromatin dynamics in the regulation of cell fate allocation during early embryogenesis. Nat. Rev. Mol. Cell Biol. 15, 723-734.

Carpenter, B. S., Lee, T. W., Plott, C. F., Rodriguez, J. D., Brockett, J. S., Myrick, D. A. and Katz, D. J. (2021). C. elegans establishes germline versus soma by balancing inherited histone methylation. Development 148, dev196600.

Chen, E. Y., Tan, C. M., Kou, Y., Duan, Q., Wang, Z., Meirelles, G. V., Clark, N. R. and Ma'ayan, A. (2013). Enrichr: interactive and collaborative HTML5 gene list enrichment analysis tool. BMC Bioinformatics 14, 128. 
Chong, J. X., Yu, J.-H., Lorentzen, P., Park, K. M., Jamal, S. M., Tabor, H. K., Rauch, A., Saenz, M. S., Boltshauser, E., Patterson, K. E., et al. (2016). Gene discovery for Mendelian conditions via social networking: de novo variants in KDM1A cause developmental delay and distinctive facial features. Genet. Med. 18, 788-795.

Coleman, R. T. and Struhl, G. (2017). Causal role for inheritance of H3K27me3 in maintaining the OFF state of a Drosophila HOX gene. Science 356.

Domanitskaya, E. and Schüpbach, T. (2012). CoREST acts as a positive regulator of Notch signaling in the follicle cells of Drosophila melanogaster. J. Cell. Sci. 125, 399-410.

Eimer, S., Lakowski, B., Donhauser, R. and Baumeister, R. (2002). Loss of spr-5 bypasses the requirement for the C.elegans presenilin sel-12 by derepressing hop-1. EMBO J. 21, 5787-5796.

Eliazer, S., Shalaby, N. A. and Buszczak, M. (2011). Loss of lysine-specific demethylase 1 nonautonomously causes stem cell tumors in the Drosophila ovary. Proc. Natl. Acad. Sci. U.S.A. 108, 7064-7069.

Engstrom, A. K., Walker, A. C., Moudgal, R. A., Myrick, D. A., Kyle, S. M., Bai, Y., Rowley, M. J. and Katz, D. J. (2020). The inhibition of LSD1 via sequestration contributes to taumediated neurodegeneration. Proc. Natl. Acad. Sci. U.S.A. 117, 29133-29143.

Eymery, A., Liu, Z., Ozonov, E. A., Stadler, M. B. and Peters, A. H. F. M. (2016). The methyltransferase Setdb1 is essential for meiosis and mitosis in mouse oocytes and early embryos. Development 143, 2767-2779.

Forneris, F., Binda, C., Adamo, A., Battaglioli, E. and Mattevi, A. (2007). Structural basis of LSD1-CoREST selectivity in histone H3 recognition. J Biol Chem 282, 20070-20074.

Foster, C. T., Dovey, O. M., Lezina, L., Luo, J. L., Gant, T. W., Barlev, N., Bradley, A. and Cowley, S. M. (2010). Lysine-specific demethylase 1 regulates the embryonic transcriptome and CoREST stability. Mol Cell Biol 30, 4851-4863.

Furuhashi, H., Takasaki, T., Rechtsteiner, A., Li, T., Kimura, H., Checchi, P. M., Strome, S. and Kelly, W. G. (2010). Trans-generational epigenetic regulation of C. elegans primordial germ cells. Epigenetics Chromatin 3, 15.

Gaydos, L. J., Wang, W. and Strome, S. (2014). Gene repression. H3K27me and PRC2 transmit a memory of repression across generations and during development. Science 345, 1515-1518.

Greer, E. L., Beese-Sims, S. E., Brookes, E., Spadafora, R., Zhu, Y., Rothbart, S. B., Aristizábal-Corrales, D., Chen, S., Badeaux, A. I., Jin, Q., et al. (2014). A histone methylation network regulates transgenerational epigenetic memory in C. elegans. Cell Rep 7, 113-126. 
Greer, E. L., Maures, T. J., Hauswirth, A. G., Green, E. M., Leeman, D. S., Maro, G. S., Han, S., Banko, M. R., Gozani, O. and Brunet, A. (2010). Members of the H3K4 trimethylation complex regulate lifespan in a germline-dependent manner in $\mathrm{C}$. elegans. Nature 466, 383-387.

Gu, Z., Eils, R. and Schlesner, M. (2016). Complex heatmaps reveal patterns and correlations in multidimensional genomic data. Bioinformatics 32, 2847-2849.

Haines, R. R., Barwick, B. G., Scharer, C. D., Majumder, P., Randall, T. D. and Boss, J. M. (2018). The Histone Demethylase LSD1 Regulates B Cell Proliferation and Plasmablast Differentiation. J Immunol 201, 2799-2811.

Hakimi, M.-A., Dong, Y., Lane, W. S., Speicher, D. W. and Shiekhattar, R. (2003). A candidate $\mathrm{X}$-linked mental retardation gene is a component of a new family of histone deacetylase-containing complexes. J Biol Chem 278, 7234-7239.

Hammoud, S. S., Nix, D. A., Zhang, H., Purwar, J., Carrell, D. T. and Cairns, B. R. (2009). Distinctive chromatin in human sperm packages genes for embryo development. Nature 460, 473-478.

Hemberger, M., Dean, W. and Reik, W. (2009). Epigenetic dynamics of stem cells and cell lineage commitment: digging Waddington's canal. Nat. Rev. Mol. Cell Biol. 10, 526-537.

Humphrey, G. W., Wang, Y., Russanova, V. R., Hirai, T., Qin, J., Nakatani, Y. and Howard, B. H. (2001). Stable histone deacetylase complexes distinguished by the presence of SANT domain proteins CoREST/kiaa0071 and Mta-L1.J Biol Chem 276, 6817-6824.

Jin, L., Hanigan, C. L., Wu, Y., Wang, W., Park, B. H., Woster, P. M. and Casero, R. A. (2013). Loss of LSD1 (lysine-specific demethylase 1) suppresses growth and alters gene expression of human colon cancer cells in a p53 - and DNMT1(DNA methyltransferase 1)-independent manner. Biochem J 449, 459-468.

Kaneshiro, K. R., Rechtsteiner, A. and Strome, S. (2019). Sperm-inherited H3K27me3 impacts offspring transcription and development in C. elegans. Nat Commun 10, 1271.

Katz, D. J., Edwards, T. M., Reinke, V. and Kelly, W. G. (2009). A C. elegans LSD1 demethylase contributes to germline immortality by reprogramming epigenetic memory. Cell 137, 308-320.

Kerenyi, M. A., Shao, Z., Hsu, Y.-J., Guo, G., Luc, S., O'Brien, K., Fujiwara, Y., Peng, C., Nguyen, M. and Orkin, S. H. (2013). Histone demethylase Lsd1 represses hematopoietic stem and progenitor cell signatures during blood cell maturation. Elife 2, e00633. 
Kerr, S. C., Ruppersburg, C. C., Francis, J. W. and Katz, D. J. (2014). SPR-5 and MET-2 function cooperatively to reestablish an epigenetic ground state during passage through the germ line. Proc. Natl. Acad. Sci. U.S.A. 111, 9509-9514.

Kim, D., Langmead, B. and Salzberg, S. L. (2015a). HISAT: a fast spliced aligner with low memory requirements. Nat. Methods 12, 357-360.

Kim, H.-M., Beese-Sims, S. E. and Colaiácovo, M. P. (2018). Fanconi Anemia FANCM/FNCM-1 and FANCD2/FCD-2 Are Required for Maintaining Histone Methylation Levels and Interact with the Histone Demethylase LSD1/SPR-5 in Caenorhabditis elegans. Genetics 209, 409-423.

Kim, J., Singh, A. K., Takata, Y., Lin, K., Shen, J., Lu, Y., Kerenyi, M. A., Orkin, S. H. and Chen, T. (2015b). LSD1 is essential for oocyte meiotic progression by regulating CDC25B expression in mice. Nat Commun 6, 10116-14.

Kim, J., Zhao, H., Dan, J., Kim, S., Hardikar, S., Hollowell, D., Lin, K., Lu, Y., Takata, Y., Shen, J., et al. (2016). Maternal Setdb1 Is Required for Meiotic Progression and Preimplantation Development in Mouse. PLoS Genet. 12, e1005970.

Kuleshov, M. V., Jones, M. R., Rouillard, A. D., Fernandez, N. F., Duan, Q., Wang, Z., Koplev, S., Jenkins, S. L., Jagodnik, K. M., Lachmann, A., et al. (2016). Enrichr: a comprehensive gene set enrichment analysis web server 2016 update. Nucleic Acids Res. 44, W90-7.

Lambrot, R., Lafleur, C. and Kimmins, S. (2015). The histone demethylase KDM1A is essential for the maintenance and differentiation of spermatogonial stem cells and progenitors. FASEB J 29, 4402-4416.

Lee, M. G., Wynder, C., Cooch, N. and Shiekhattar, R. (2005). An essential role for CoREST in nucleosomal histone 3 lysine 4 demethylation. Nature 437, 432-435.

Lee, M.-C. and Spradling, A. C. (2014). The progenitor state is maintained by lysinespecific demethylase 1-mediated epigenetic plasticity during Drosophila follicle cell development. Genes Dev. 28, 2739-2749.

Lee, T. W.-S., David, H. S., Engstrom, A. K., Carpenter, B. S. and Katz, D. J. (2019). Repressive H3K9me2 protects lifespan against the transgenerational burden of COMPASS activity in C. elegans. elifesciences.org

8, 502 .

Li, E. (2002). Chromatin modification and epigenetic reprogramming in mammalian development. Nat Rev Genet 3, 662-673.

Liao, Y., Smyth, G. K. and Shi, W. (2014). featureCounts: an efficient general purpose program for assigning sequence reads to genomic features. Bioinformatics 30, 923-930. 
Love, M. I., Huber, W. and Anders, S. (2014). Moderated estimation of fold change and dispersion for RNA-seq data with DESeq2. Genome Biol. 15, 550.

Ma, P., Pan, H., Montgomery, R. L., Olson, E. N. and Schultz, R. M. (2012). Compensatory functions of histone deacetylase 1 (HDAC1) and HDAC2 regulate transcription and apoptosis during mouse oocyte development. Proc. Natl. Acad. Sci. U.S.A. 109, E481-9.

Mačinković, I., Theofel, I., Hundertmark, T., Kovač, K., Awe, S., Lenz, J., Forné, I., Lamp, B., Nist, A., Imhof, A., et al. (2019). Distinct CoREST complexes act in a cell-typespecific manner. Nucleic Acids Res. 47, 11649-11666.

Moehrle, A. and Paro, R. (1994). Spreading the silence: epigenetic transcriptional regulation during Drosophila development. Dev Genet 15, 478-484.

Morgan, H. D., Santos, F., Green, K., Dean, W. and Reik, W. (2005). Epigenetic reprogramming in mammals. Hum Mol Genet 14 Spec No 1, R47-58.

Myrick, D. A., Christopher, M. A., Scott, A. M., Simon, A. K., Donlin-Asp, P. G., Kelly, W. G. and Katz, D. J. (2017). KDM1A/LSD1 regulates the differentiation and maintenance of spermatogonia in mice. PLOS ONE 12, e0177473.

Nicholson, T. B., Singh, A. K., Su, H., Hevi, S., Wang, J., Bajko, J., Li, M., Valdez, R., Goetschkes, M., Capodieci, P., et al. (2013). A hypomorphic lsd1 allele results in heart development defects in mice. PLoS ONE 8, e60913.

Rechtsteiner, A., Ercan, S., Takasaki, T., Phippen, T. M., Egelhofer, T. A., Wang, W., Kimura, H., Lieb, J. D. and Strome, S. (2010). The histone H3K36 methyltransferase MES-4 acts epigenetically to transmit the memory of germline gene expression to progeny. PLoS Genet. 6, e1001091.

Seisenberger, S., Andrews, S., Krueger, F., Arand, J., Walter, J., Santos, F., Popp, C., Thienpont, B., Dean, W. and Reik, W. (2012). The dynamics of genome-wide DNA methylation reprogramming in mouse primordial germ cells. Mol. Cell 48, 849-862.

Shi, Y., Lan, F., Matson, C., Mulligan, P., Whetstine, J. R., Cole, P. A., Casero, R. A. and Shi, Y. (2004). Histone demethylation mediated by the nuclear amine oxidase homolog LSD1. Cell 119, 941-953.

Shi, Y., Sawada, J.-I., Sui, G., Affar, E. B., Whetstine, J. R., Lan, F., Ogawa, H., Luke, M. P.S., Nakatani, Y. and Shi, Y. (2003). Coordinated histone modifications mediated by a CtBP co-repressor complex. Nature 422, 735-738.

Shi, Y.-J., Matson, C., Lan, F., Iwase, S., Baba, T. and Shi, Y. (2005). Regulation of LSD1 histone demethylase activity by its associated factors. Mol. Cell 19, 857-864. 
Simon, J. A. and Tamkun, J. W. (2002). Programming off and on states in chromatin: mechanisms of Polycomb and trithorax group complexes. Curr Opin Genet Dev 12, 210218.

Stavropoulos, P., Blobel, G. and Hoelz, A. (2006). Crystal structure and mechanism of human lysine-specific demethylase-1. Nat Struct Mol Biol 13, 626-632.

Tabuchi, T. M., Rechtsteiner, A., Jeffers, T. E., Egelhofer, T. A., Murphy, C. T. and Strome, S. (2018). Caenorhabditis elegans sperm carry a histone-based epigenetic memory of both spermatogenesis and oogenesis. Nat Commun 9, 4310.

Tosic, M., Allen, A., Willmann, D., Lepper, C., Kim, J., Duteil, D. and Schüle, R. (2018). Lsd1 regulates skeletal muscle regeneration and directs the fate of satellite cells. Nat Commun 9, 366.

Tunovic, S., Barkovich, J., Sherr, E. H. and Slavotinek, A. M. (2014). De novo ANKRD11 and KDM1A gene mutations in a male with features of KBG syndrome and Kabuki syndrome. Am. J. Med. Genet. A 164A, 1744-1749.

Wang, J., Hevi, S., Kurash, J. K., Lei, H., Gay, F., Bajko, J., Su, H., Sun, W., Chang, H., Xu, G., et al. (2009). The lysine demethylase LSD1 (KDM1) is required for maintenance of global DNA methylation. Nat. Genet. 41, 125-129.

Wang, J., Scully, K., Zhu, X., Cai, L., Zhang, J., Prefontaine, G. G., Krones, A., Ohgi, K. A., Zhu, P., Garcia-Bassets, I., et al. (2007). Opposing LSD1 complexes function in developmental gene activation and repression programmes. Nature 446, 882-887.

Wasson, J. A., Simon, A. K., Myrick, D. A., Wolf, G., Driscoll, S., Pfaff, S. L., Macfarlan, T. S. and Katz, D. J. (2016). Maternally provided LSD1/KDM1A enables the maternal-tozygotic transition and prevents defects that manifest postnatally. Elife 5, 296.

Wen, C., Levitan, D., Li, X. and Greenwald, I. (2000). spr-2, a suppressor of the egg-laying defect caused by loss of sel-12 presenilin in Caenorhabditiselegans, is a member of the SET protein subfamily. PNAS 97, 14524-14529.

Wingett, S. W. and Andrews, S. (2018). FastQ Screen: A tool for multi-genome mapping and quality control. F1000Res 7, 1338.

Wu, S.-F., Zhang, H. and Cairns, B. R. (2011). Genes for embryo development are packaged in blocks of multivalent chromatin in zebrafish sperm. Genome Res 21, 578589.

Yang, M., Gocke, C. B., Luo, X., Borek, D., Tomchick, D. R., Machius, M., Otwinowski, Z. and Yu, H. (2006). Structural basis for CoREST-dependent demethylation of nucleosomes by the human LSD1 histone demethylase. Mol. Cell 23, 377-387. 
Yao, H., Goldman, D. C., Nechiporuk, T., Kawane, S., McWeeney, S. K., Tyner, J. W., Fan, G., Kerenyi, M. A., Orkin, S. H., Fleming, W. H., et al. (2014). Corepressor Rcor1 is essential for murine erythropoiesis. Blood 123, 3175-3184.

You, A., Tong, J. K., Grozinger, C. M. and Schreiber, S. L. (2001). CoREST is an integral component of the CoREST- human histone deacetylase complex. PNAS 98, 1454-1458.

Zheng, H., Huang, B., Zhang, B., Xiang, Y., Du, Z., Xu, Q., Li, Y., Wang, Q., Ma, J., Peng, X., et al. (2016). Resetting Epigenetic Memory by Reprogramming of Histone Modifications in Mammals. Mol. Cell 63, 1066-1079.

Zhu, D., Hölz, S., Metzger, E., Pavlovic, M., Jandausch, A., Jilg, C., Galgoczy, P., Herz, C., Moser, M., Metzger, D., et al. (2014). Lysine-specific demethylase 1 regulates differentiation onset and migration of trophoblast stem cells. Nat Commun 5, 3174-14.

Zhu, W., Xu, X., Wang, X. and Liu, J. (2019). Reprogramming histone modification patterns to coordinate gene expression in early zebrafish embryos. BMC Genomics 20, 248. 